Ann. Génét. Sél. anim., 1976, 8 (2), 233-264.

\title{
UTILISATION DES RACES RUSTIQUES EN CROISEMENT AVEC DES RACES A VIANDE : EXEMPLE DE LA RACE GASCONNE (1)
}

\author{
B. BIBÉ, J. FRÉBLING, F. MÉNISSIER et B. VISSAC \\ avec la collaboration technique de Michèle Briend, J.-J. Chambeyron, \\ G. P. Fabre, P. Gillard, P. Leroyer \\ Station de Génétique quantitative et appliquée, \\ Centre national de Recherches zootechniques, I. N. R. A., \\ 78350 Jouy en Josas (France)
}

\section{RÉSUMÉ}

L'étude concerne la race Gasconne utilisée dans divers systèmes de croisements, et complète des résultats antérieurs obtenus avec d'autres races rustiques. Le troupeau expérimental, exploité durant 3 années dans diverses localisations, était constitué au départ d'une centaine de génisses provenant de 5 types génétiques : Gascon (GG), Charolais (CC), Blond d'Aquitaine (BB), Charolais $\times$ Gascon (CG) et Blond $\times$ Gascon $(\mathrm{BG})$. Ces femelles étaient conduites en croisement terminal avec des taureaux Charolais et Blonds d'Aquitaine culards. Tous leurs veaux mâles et femelles, après sevrage vers 7 mois, ont été engraissés avec un contrôle individuel de leurs performances; ils ont été abattus à l'âge de $\mathrm{I}_{4}$ mois $\mathrm{I} / 2$ pour les mâles alors que les femelles ne l'ont été qu'à 2 I mois après deux phases d'engraissement séparées par une période de pâturage.

Le schéma a permis d'étudier l'incidence de l'augmentation du pourcentage de gènes de races à viande sur la production de viande prise dans son ensemble (productivités pondérale et numérique). Cette augmentation portait sur les produits (50 p. Ioo à Ioo p. Ioo) et sur les mères (o p. roo à roo p. Ioo). En outre, il a été possible de comparer, à ces deux niveaux (mères et produits), les deux races paternelles utilisées pour obtenir cette augmentation.

Il ressort de cette expérience que l'augmentation du pourcentage de gènes de races à viande, a :

- un effet positif sur la marge brute (valeur à la vente moins frais d'alimentation) réalisée par produit engraissé ( $14 \mathrm{p}$. I oo de supplément pour les animaux à $75 \mathrm{p}$. I oo ou Ioo p. Ioo de gènes de race à viande par rapport aux animaux à $50 \mathrm{p}$. Ioo de ces mêmes gènes) et sur la valeur de réforme des femelles (23 p. Ioo et 32 p. roo de supplément sur les vaches gasconnes pures, respectivement pour les femelles croisées et les femelles de races à viande ;

- un effet défavorable sur la productivité numérique (taux de veaux sevrés par femelle mise à la reproduction), effet qui réduit, voire annule, celui favorable sur la croissance musculaire : la supériorité relative sur les femelles gasconnes, des vaches blondes d'Aquitaine et croisées blondes $\times$ gasconnes est voisine đe ro p. roo; celle obtenue avec des vaches charolaises ou croisées charolaises est voisine de $o$.

(1) Cette étude a été réalisée avec le soutien financier du Ministère de l'Agriculture (C.E.R.A.F.E.R. de Toulouse) et grâce à la collaboration de la Coopérative d'Élevage et d'Insémination artificielle du SudOuest (Coopelso à Soual, 8 I I Io Dourgne) et des Stations d'Élevage des Ruminants et de Technologie de la Viande du C.R.V.Z. (I.N.R.A., Theix, 631 10 Beaumont). 
$\mathrm{Si}$, de plus, on prend en compte les besoins d'entretien des femelles, besoins qui sont supérieurs pour les femelles croisées ou de race pure à viande en raison de leur format plus élevé, les différences de productivité globale sont à nouveau réduites entre les femelles croisées ou de race pure blonde d'Aquitaine et les femelles gasconnes, les femelles croisées ou de race pure charolaise ayant quant à elles une productivité inférieure à celle des gasconnes.

Avec les réserves liées à ces paramètres, l'absorption de la race Gasconne par une race à viande ne se justifie pas. L'exploitation des mères croisées, en croisement terminal, est certainement la solution la plus avantageuse ; leur renouvellement étant assuré soit par croisement alternatif, soit par maintien d'un noyau de Gasconnes en race pure. Il est difficile de généraliser ces conclusions établies sur un échantillon trop restreint et exploité dans des conditions particulières. Des recherches plus globales sont nécessaires pour préciser les conditions optimales d'exploitation des zones difficiles par les races rustiques.

\section{INTRODUCTION}

La rentabilité de la production de viande obtenue à partir d'un troupeau de vaches allaitantes, dépend de nombreux caractères déterminés séparément ou conjointement par le génotype des parents, de la mère en particulier, et par celui du veau (DICKERSON, I970 ; CARTWRIGHT, I970 ; VISSAC, I972). En théorie, l'amélioration de cette rentabilité pourrait être obtenue en maximisant l'ensemble de ces caractères (CunNingham, 1973). En pratique, le choix et la sélection des races ont surtout porté sur la croissance musculaire (poids à un âge donné et conformation) c'est-à-dire sur des caractères à héritabilité moyenne ou forte; ils ont entrainé en moyenne, d'une race à l'autre ou à l'intérieur des races (MÉNISSIER et al., I974; FoulLEY et MéNIssIER, 1974), une réduction de la fertilité, de la viabilité, des aptitudes maternelles propres et des facultés d'adaptation (" fitness ").

Les premiers caractères dépendant plutôt du veau et les seconds des parents, de la mère surtout, on est donc conduit à rechercher des systèmes de croisement dans lesquels la constitution génétique et les objectifs de sélection différeraient entre la mère et le veau (MÉNISSIER et al., I974). On est bien sûr limité dans cette voie par la faible fertilité de la femelle bovine tant que les techniques d'inovulation ne seront pas opérationnelles. En outre, la pratique du croisement permet de bénéficier des avantages liés à la vigueur hybride, avantages qui se manifestent essentiellement pour le deuxième groupe de caractères et qui concernent à la fois le génotype de la mère et celui du veau.

Les races rustiques à fins multiples (lait, viande, travail) exploitées dans les zones de montagnes et dans les régions subhumides du Bassin méditerranéen constituent précisément des populations femelles sélectionnées pour la fertilité et les aptitudes maternelles, mais aussi bien adaptées à leur milieu. Depuis 1'abandon de la traite et de la traction animale, elles sont disponibles pour une exploitation en troupeaux de femelles allaitantes conduites en croisement avec des taureaux de race à viande à fort développement musculaire. L'effectif de ces femelles diminuant rapidement, la recherche de systèmes de croisement avec des mâles à viande doit se situer dans l'optique plus large d'une valorisation optimale en système extensif des ressources naturelles par ces races en voie de dépeuplement.

Une première expérience a été réalisée en station expérimentale à partir de 
femelles de race d'Aubrac, en vue de comparer l'efficacité respective de l'élevage en race pure, du croisement de première génération et du croisement à double étage avec la conservation des femelles croisées $F_{1}$ pour la reproduction (VISSAC, I970; BIBÉ et al., I974). Cette dernière formule permet de limiter la fraction de population maintenue en race pure, fraction qui, dans le cas de la race d'Aubrac par exemple, ne parait plus suffisante aujourd'hui pour assurer le renouvellement dans l'optique d'une organisation de l'élevage en vue d'un strict croisement de première génération. Dans cet article nous nous référerons souvent aux principaux résultats de cette expérience en race $A u b r a c$ sans en rappeler chaque fois la référence bibliographique. Une expérience similaire a été entreprise en Sardaigne en vue de comparer les mêmes systèmes de croisement dans les conditions de l'élevage extensif en montagne (CASU, et al., I975) ; le cheptel femelle de départ était constitué des deux races locales de format très différent (la Sarde et la Modicane) et de la Brune des Alpes disponible dans les élevages laitiers des zones de plaines avoisinantes.

La présente étude vise à compléter les travaux précédents sur plusieurs points. Elle concerne la race Gasconne qui représente environ 35 ooo des 45 ooo femelles allaitantes de la zone des Pyrénées centrales. Ces femelles sont exploitées pour partie dans des troupeaux sédentaires pour partie suivant une transhumance saisonnière dans des estives de haute montagne. Les troupeaux pratiquent surtout la monte naturelle; il n'y a que Io 000 à I5 000 inséminations artificielles par an en race Gasconne. Outre le croisement de première génération et le croisement à double étage, l'introduction des échantillons de femelles de races à viande dans cette étude permet donc d'envisager la situation limite du croisement d'absorption de la race locale par des races à viande.

\section{MATÉRIEL ANIMAL, CONDUITE DE L'EXPÉRIENCE E'T MÉTHODE D'ANALYSE}

\section{I. - Types génétiques et schéma d'accouplement}

L'expérience a porté sur ror génisses contemporaines appartenant aux cinq types génétiques suivants (race paternelle $\times$ race maternelle) :

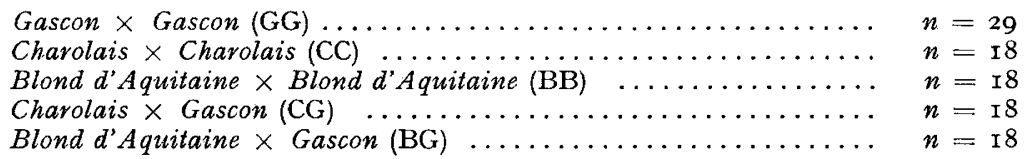

Elles étaient, pour la plupart, originaires d'élevages du Sud-Ouest pratiquant l'insémination artificielle mais, contrairement à l' "expérience $A u b r a c$ ", leurs pères étaient de phénotype inconnu en ce qui concerne le caractère culard. Les femelles de races à viande étaient toutes de phénotype normal.

Chaque lot était inséminé au cours de 3 années consécutives $\left(\mathrm{I}^{\mathrm{er}}, 2^{\mathrm{e}}\right.$ et $3^{\mathrm{e}}$ vêlages) pour moitié avec deux taureaux Charolais, pour moitié avec deux taureaux Blonds d'Aquitaine, avec alternance de la race paternelle d'une année sur l'autre. Compte tenu de la fréquence élevée des difficultés de vêlage dans l'expérience similaire entreprise sur génisses $A u b r a c$ et croisées, ces taureaux ont été choisis après contrôle de descendance parmi ceux à musculature hypertrophiée qui fournissaient des veaux plus légers à la naissance. 


\section{2. - Conduite des animaux et variables étudiées}

a) Femelles reproductrices.

Ces femelles sont nées au cours du I er trimestre 1968 mais les dates exactes de naissance n'étaient connues que pour une fraction de l'échantillon. Elles sont issues d'élevages à viande extensifs pratiquant le sevrage à 6-7 mois. Seules les Blondes d'Aquitaine (BB) proviennent de troupeaux pratiquant la production de veaux de boucherie ; elles ont dû, pour les besoins de cette expérience être sevrées précocement à 3-4 mois après une phase d'allaitement intensif. Ces animaux ont été rassemblés au sevrage dans une première localisation (Soual). En raison de contraintes matérielles, ces animaux ont dû être ensuite déplacés plusieurs fois (4) au cours de l'expérience (tabl. I). Ils étaient conduits en zéro-pâturage tout au long de l'année, sur une aire d'extension suffisante pour qu'on puisse apprécier leur comportement en liberté. Ils ont reçu dès le sevrage et durant le premier hiver un régime semi-intensif $(6,5 \mathrm{~kg}$ de foin et $2,3 \mathrm{~kg}$ de concentré par femelle et par jour) destiné à obtenir une manifestation précoce de la sexualité en vue d'un premier vêlage à ' 2 ans. Ensuite l'alimentation a été à base de foin ou de fourrages verts, excepté au cours de deux périodes : mars-avril r9jo et fin août début septembre de la même année, où un apport de concentré a été fourni pour subvenir aux besoins supérieurs des génisses en fin de gestation et pour pallier un déficit saisonnier de production fourragère particulièrement accusé (sècheresse estivale persistante).

La mise à la reproduction a été pratiquée en 1969 et 1970 après synchronisation d'œestrus pour les femelles cyclées, et induction artificielle d'ovulation pour les femelles non cyclées. En I969, ces traitements ont été effectués à date fixe pour tout le troupeau ; les inséminations ont eu lieu sur chaleurs induites ( 2 juillet r969) et au cours d'une période de 8 semaines consécutives. Elles ont été suivies d'une "repasse " de 6 semaines avec un taureau Blond d'Aquitaine. Les génisses grestantes à la suite de cette dernière période n'ont pas été prises en considération pour les campagnes ultérieures de reproduction. En 1970, les femelles ont été traitées en deux lots suivant leur date de vêlage : les inséminations ont été réalisées sur chaleurs induites les 7 juillet 1970 ( ${ }^{\text {er }}$ lot) et 22 juillet 1970 ( $2^{\mathrm{e}}$ lot), puis jusqu'au I er septembre 1970 c'est-à-dire pendant 5 à 7 semaines. Les chaleurs ont été contrôlées biquotidiennement avec trois taureaux vasectomisés dès l'âge de Io mois chez les génisses en I969, dès le vêlage en I970 et, pour ces deux années, jusqu'à la fin de la période d'insémination. Les femelles non gestantes à la fin de cette période étaient éliminées de l'expérience. I.es contrôles de reproduction relatifs aux femelles encore disponibles en ${ }^{9} 97$ en vue du $3^{\mathrm{e}}$ vêlage à 4 ans, terme de l'expérience, n'ont pas été considérés. Néanmoins les veaux issus de ces femelles ont été contrôlés et leurs performances utilisées dans l'analyse.

Les vêlages des génisses ont été répartis en trois catégories selon la difficulté d'extraction du fœtus :

$\mathbf{I}=$ facile : sans assistance ou avec l'aide d'une seule personne

$2=$ difficile : nécessitant l'aide de deux à trois personnes ou l'utilisation d'une vêleuse ;

3 = césarienne ou embryotomie : extraction par voie chirurgicale.

En plus de cette note d'aptitude au vêlage attribuée à chaque mise bas, nous avons analysé les composantes morphologiques de l'aptitude au vêlage à partir des contrôles suivants :

- mensurations externes corporelles et internes de l'ouverture pelvienne (MÉnIsSIER et VISSAC, I97I) pour les femelles à un an et à deux ans après vêlage ;

- mensurations externes du veau, placé dans sa position lors de la mise bas, au cours des 48 heures qui suivent la naissance.

La préparation des femelles à la mise bas a été appréciée de façon subjective au niveau de la vulve, de la mamelle et des ligaments sacro-sciatiques; on a enfin noté, lors des mises bas, les points éventuels de résistance à l'expulsion du fœetus chez la mère et le veau.

Les résultats relatifs au vêlage ont donné lieu séparément à une première analyse (ABDALLAH, I97. ; ABDAllaH et al., 197 I $a$ et $b$ ).

Les femelles ont été pesées à intervalles réguliers ainsi qu'après vêlage en 1970 . Cette même année des contrôles mensuels du poids ont eu lieu au cours de l'été, lorsque le troupeau a subi une importante restriction alimentaire du fait de la sécheresse estivale. Deux contrôles laitiers ont été réalisés à date fixe sur ces femelles (13 mai et 27 juin 1970) par pesée des veaux avant et après tétée ; l'âge moyen des veaux était alors respectivement de 3 et 7 semaines. Ces observations ont été complétées par un contrôle continu des tétées effectuées par les veaux entre $5 \mathrm{~h} 30$ le matin et 20 heures le soir, pendant deux jours consécutifs ( 12 et I 3 septembre I970). Les veaux étaient alors âgés de 4 mois $\mathrm{I} / 2$ à 5 mois.

Au cours de cette période estivale de 1970 , nous avons effectué du 20 au 25 juillet inclus et du 18 au 22 août inclus un contrôle du comportement alimentaire (abreuvement, consommation, 


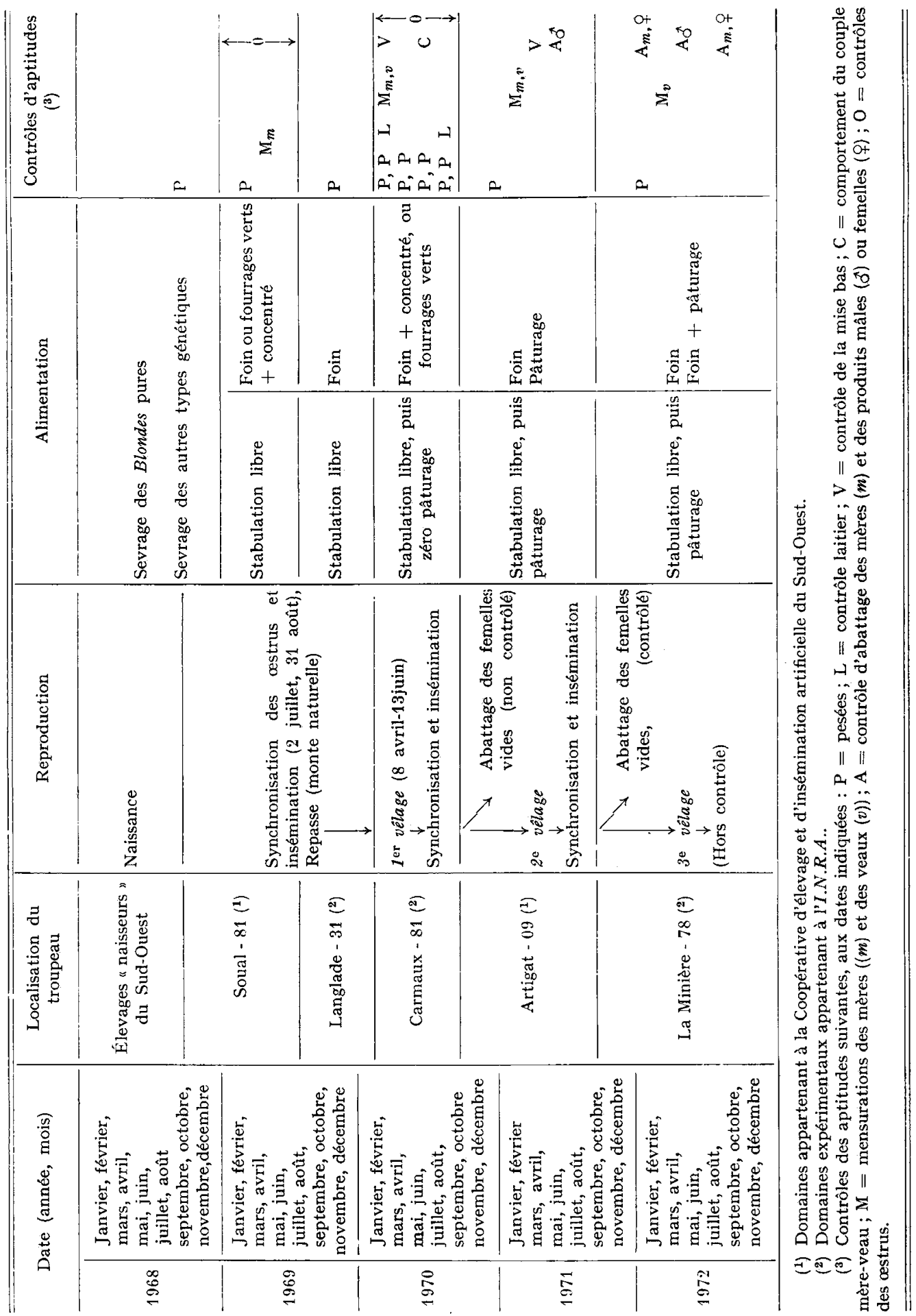


rumination, repos) du troupeau soumis au rythme d'alimentation suivant : apport de fourrages verts à l'auge entre $8 \mathrm{~h}$ zo et 9 heures, apport de foin, de paille et éventuellement de concentré entre I 5 heures et 16 heures. Cette distribution de fourrage était effectuée sur une surface suffisamment vaste pour limiter les compétitions entre animaux. Le troupeau ainsi contrôlé comportait 67 vaches et 58 veaux. Ce contrôle était réalisé par observation du comportement de chaque vache du troupeau toutes les demi-heures. Toutefois, étant donné l'insuffisance de ce rythme de contrôle pour estimer la fréquence d'abreuvement, nous avons procédé à un contrôle permanent du comportement des animaux à cet égard entre 6 heures et 2 I heures, les I I et I 3 août.

Vers la fin de l'année 1971, nous avons procédé à un engraissement, durant 2 mois environ, des femelles non gestantes après le deuxième vêlage, puis à un contrôle d'abattage et de carcasse. Le rendement a été estimé par le rapport du poids de la carcasse froide au poids vif vide obtenu en déduisant du poids avant abattage le contenu du tube digestif (estomac et intestins). Les dépôts adipeux du péritoine, du mésentère et périrénaux ont été pesés. La composition de la carcasse a été estimée par dissection du morceau unicostal (I I e côte) selon la technique décrite par Geay et Béranger (I969).

\section{b) Produits mâles et femelles.}

Les veaux mâles et femelles issus des trois premiers vêlages ont été contrôlés et élevés jusqu'à l'abattage, suivaut une technique standard. Le sevrage est intervenu à 7 mois. Les veaux recevaient ensuite du foin à volonté puis, à partir de 7 mois $1 / 2$, une quantité croissante d'un concentré comportant $2 / 3$ de luzerne déshydratée et $I / 3$ de pulpe déshydratée. La distribution de foin était supprimée à partir du $8^{\mathrm{e}}$ mois où commençait le contrôle de la quantité consommée (ad libitum). L'engraissement a eu lieu en stabulation entravée.

Les mâles étaient abattus après 182 jours d'engraissement ( 14 mois et demi). Les femelles étaient contrôlées de la même façon pendant les 98 premiers jours (phase I) puis élevées au pâturage et engraissées pendant 84 jours avant abattage avec le même concentré (phase 2); leur abattage intervenait à environ $2 \mathrm{I}$ mois. La quantité de concentré consommée (ad libitum) était contrôlée chaque jour pendant la (ou les) phase (s) d'engraissement. Les animaux étaient pesés tous les I 4 jours. Un contrôle objectif de la morphologie par mesures corporelles externes et de l'ouverture pelvienne était effectué à environ 13 mois pour les mâles et I 2 mois pour les femelles. On pratiquait également à cet âge une observation du comportement des animaux sous l'effet d'un stress thermique de 12 heures : les animaux étaient placés dans une chambre expérimentale chauffée durant la journée jusqu'à $37^{\circ}$; cette température était maintenue pendant 5 heures. Toutes les demi-heures leur température rectale, rythme cardiaque, rythme respiratoire et taux de sudation étaient enregistrés. Comme pour l'aptitude au vêlage, cette information a été analysée séparément (VISINE, I973).

Le rendement à l'abattage ainsi que la composition de la carcasse étaient estimés selon la même technique que pour les vaches de réforme (voir ci-dessus). En outre, nous avons effectué des mensurations de la carcasse visant à déterminer les principales dimensions squelettiques (longueur du tronc : du milieu de la I $^{\text {re }}$ côte à la base de la symphyse pubienne, et longueur du membre postérieur : de la symphyse pubienne au bord interne de la section du jarret) et épaisseurs des plans musculaires au niveau du faux-filet et de la cuisse (Frébling et al., I967). Enfin un contrôle des qualités de la viande a été fait sur l'échantillon du muscle long dorsal prélevé après dissection du morceau unicostal ; les mesures concernaient : la dureté de la viande $\left(\mathrm{kg} \mathrm{f} / \mathrm{cm}^{2}\right)$, le $\mathrm{pH}$, le taux de pertes en eau (p. roo), la quantité de fer héminique (mg/g). Leur signification et leur mode de réalisation sont décrits par ailleurs (Jounné et al., I973).

\section{3. - Méthode d'analyse}

Pour les critères liés exclusivement au génotype maternel et pour ceux concernant la productivité numérique (fertilité et aptitude au vêlage), l'analyse a été limitée à une comparaison des moyennes relatives à chaque type génétique.

Pour les critères relatifs aux veaux, nous avons estimé par moindres-carrés les effets de l'année cle naissance (confondue avec l'âge des mères), de la race paternelle, du type génétique maternel et du sexe, dans le cas d'un modèle sans interaction ; l'existence d'une interaction globale au niveau du modèle était néanmoins testée. Toutefois, en ce qui concerne les critères relatifs à la phase d'engraissement et à l'abattage, l'analyse des données a été réalisée séparément pour les veaux mâles et femelles. Les estimées relatives aux divers effets des niveaux des facteurs, les valeurs de F correspondant à chaque type d'effet et à l'interaction globale ainsi que l'écart-type résiduel (intra-cellule), figurent pour chaque variable sur les tableaux 3, 5, 6, 7, 8, 9, Io et II.

A partir des estimées relatives aux races paternelles $\left(\mathrm{P}_{c h}=\right.$ Charolaise, $\mathbf{P}_{b l}=$ Blonde $d^{\prime}$ Aquitaine) et aux types génétiques maternels $\left(\mathbf{M}_{c h}=\right.$ Charolais, $\mathbf{M}_{b l}=$ Blond d'Aquitaine, 
$\mathbf{M}_{\times c h}$ et $\mathbf{M}_{\times b l}=$ croisés $G a s c o n s$ avec respectivement les deux races à viande précédentes, $\mathbf{M}_{g a}$ $=$ Gascon) des veaux, nous avons comparé les deux races à viande (Charolaise/Blonde d'Aquitaine) du point de vue de leurs effets directs et maternels sur les caractères influencés simultanément par ces deux types d'effets (Dickerson 1969). En supposant les effets d'hétérosis identiques quelle que soit la race paternelle utilisée, nous avons en effet :

(I) Effet Direct (Charolais/Blond d'Aquitaine) $=2 \times\left(\mathrm{P}_{c h}-\mathrm{P}_{b l}\right)$

(2) Effet Maternel (Charolais/Blond d'Aquitaine $)=2 \times\left(\mathbf{M}_{\times c h}-\mathbf{M}_{x b l}\right)-\left(\mathrm{P}_{c h}-\mathrm{P}_{b l}\right)$

(3) Effet Maternel (Charolais/Blond d'Aquitaine $)=\left(\mathbf{M}_{c h}-\mathrm{M}_{b l}\right)-\left(\mathrm{P}_{c h}-\mathbf{P}_{b l}\right)$

Nous obtenons deux estimations des effets maternels selon que l'on compare les mères de race pure (3) ou les croisées (2).

Le schéma utilisé permettait aussi de déterminer les effets non additifs des gènes sur les effets maternels, à partir des estimées ci-dessus relatives aux veaux des mères croisées et de race pure. Pour chacune des races à viande nous avons :

(4) Hétérosis sur l'effet maternel (Charolais) $=\mathrm{M}_{\times c h}-\frac{\left(\mathrm{M}_{g a}+\mathrm{M}_{c h}\right)}{2}$

(5) Hétérosis sur l'effet maternel (Blonde d'Aquitaine $)=\mathrm{M}_{\times b l}-\frac{\left(\mathrm{M}_{g a}+\mathrm{M}_{b l}\right)}{2}$

Pour chacune de ces 5 estimations, les résultats ont été exprimés soit en unités d'écarttype résiduel ((I) (2) et (3)), soit pour les deux dernières ((4) et (5)) en p. Ioo de la moyenne des races parentales.

Nous avons enfin tenté de réaliser un bilan économique global au niveau des races paternelles et des types génétiques maternels envisagés séparément dans ce but. Nous avons considéré les critères suivants :

- Bilan par veau en engraissement ou valorisation du veau sevré $\left(\mathrm{B}_{\mathrm{snimale}}\right)$ : c'est la différence entre la valeur à la vente (estimée par le produit : poids de carcasse $\times p$. Ioo de muscle du morceau unicostal $\times I_{4} F$ ) et le coat de l'alimentation pendant la phase d'engraissement (estimé par le produit : consommation totale $\times 0,4 \mathrm{~F}$ ). Les paramètres économiques ci-dessus sont les mêmes que ceux utilisés dans l' "expérience Aubrac ", afin de faciliter la comparaison des résultats des deux études.

- Bilan par femelle gestante ( $\left.B_{\text {gestante}}\right)$ pour les types génétiques maternels utilisés. $\mathrm{Si}$, pour un type génétique maternel donné, $B$ mâle et $B$ femelle sont les valeurs précédemment obtenues respectivement pour les veaux mâles et femelles et $B$ réforme la valeur moyenne de réforme des vaches obtenue sur l'échantillon des vaches contrôlées à l'abattage, le bilan par femelle gestante, établi sur $k$ campagnes de reproduction, peut s'exprimer par :

$$
B_{\text {gestante }}=B_{\text {vache }}+k \times\left(B_{\text {mâle }}+B_{\text {femelle }}\right) / 2
$$

- Bilan par femelle mise en reproduction $\left(B_{\text {reproduction }}\right)$. En utilisant le taux de veaux sevrés par femelle mise à la reproduction $(p)$, ce bilan est égal à :

$$
B_{\text {reproduction }}=B_{\text {vache }}+k \times p \times\left(B_{\text {mâle }}+B_{\text {lemelle }}\right) / 2
$$

- Bilan par unité de fourrages grossiers $\left(B_{\text {fourrage }}\right)$. En supposant en première approximation que cette consommation ne concerne que les mères et que, pour un type génétique donné, elle est proportionnelle à leur poids moyen à l'âge de 2 ans (poids), ce bilan est proportionnel à

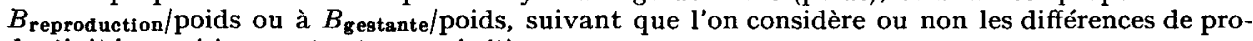
ductivité numérique entre types génétiques.

\section{RÉSULTATS ANALYTTIQUES}

Nous avons indiqué dans les tableaux qui suivent (tabl. 2 à 12) les principaux résultats obtenus lors de 1'analyse statistique. Nous limiterons nos commentaires aux différences entre types génétiques. Nous nous sommes permis de discuter des différences mêmes non significatives, lorsque celles-ci expriment des tendances déjà observées lors d'expérimentations antérieures (expérience " $A u b r a c$ ") ou contemporaines (expérience "Sarde », CASU et al., I975). De plus, nous sommes conscients de la 
nécessité qu'il y aurait à confirmer ces tendances lors d'essais ultérieurs qui, en outre, devraient être conduits dans des conditions plus ressemblantes à celles des zones de montagne.

\section{I. - Productivité numérique}

a) Précocité sexuelle (tabl. 2).

Les premières chaleurs sont apparues environ 2 mois plus tard dans cette expérience que dans l'expérience similaire réalisée à partir de femelles $A u b r a c$ élevées suivant un régime alimentaire et un niveau de croissance après sevrage comparables. De ce fait l'objectif d'un premier vêlage à 2 ans a dû être supprimé et les génisses ont été mises à la reproduction vers I6 à $\mathrm{I} 7$ mois alors que 6I p. Ioo seulement avaient manifesté des signes d'œstrus à cet âge. L,es génisses Blondes d'Aquitaine et croisées

TABLEAU 2

Performances d'élevage des femelles de différents types génétiques (Valeurs moyennes brutes)

Breeding records of the cows from various genotypes (unadjusted means)

\begin{tabular}{|c|c|c|c|c|c|c|}
\hline \multirow{2}{*}{\multicolumn{2}{|c|}{ Variable }} & \multicolumn{5}{|c|}{ Type génétique } \\
\hline & & Charolais & $\begin{array}{l}\text { Charolais } \\
\times \text { Gascon }\end{array}$ & Gascon & $\begin{array}{l}\text { Blond d'Aq. } \\
\times \text { Gascon }\end{array}$ & $\begin{array}{c}\text { Blond } \\
\text { d'Aquitaine }\end{array}$ \\
\hline 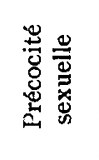 & 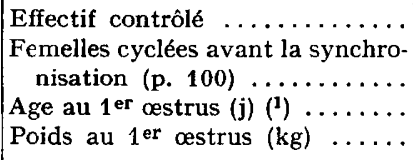 & \begin{tabular}{rl|}
\multicolumn{1}{r}{18} & $\left({ }^{3}\right)$ \\
72,2 & $(13)$ \\
402 & $(6)$ \\
353 & $(13)$
\end{tabular} & \begin{tabular}{rl|}
\multicolumn{2}{r|}{18} \\
88,9 & $(16)$ \\
403 & $(9)$ \\
360 & $(16)$
\end{tabular} & \begin{tabular}{rr|}
65,5 & $(19)$ \\
419 & $(12)$ \\
331 & $(19)$
\end{tabular} & 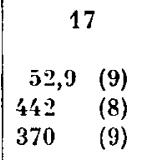 & $\begin{array}{cc} & 18 \\
22,2 & (4) \\
444 & (2) \\
350 & (4)\end{array}$ \\
\hline 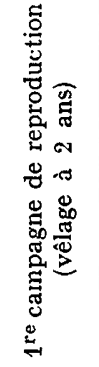 & 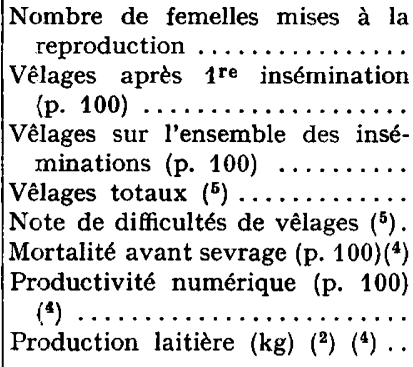 & \begin{tabular}{cc|}
18 & \\
16,7 & $(3)$ \\
& \\
50,0 & $(9)$ \\
72,2 & $(13)$ \\
$2,63(13)$ \\
22,2 & $(2)$ \\
& \\
38,9 \\
3,7
\end{tabular} & $\begin{array}{cc}18 \\
55,6 & (10) \\
& \\
77,8 & (14) \\
94,5 & (17) \\
2,31 & (17) \\
0 & (0) \\
77,8 \\
6,1\end{array}$ & $\begin{array}{cc}29 \\
48,3 & (14) \\
& \\
75,8 & (22) \\
93,1 & (27) \\
2,28(27) \\
17,4(4) \\
65,5 \\
8,7\end{array}$ & \begin{tabular}{cl}
\multicolumn{1}{c}{16} \\
$25,0 \quad(4)$ \\
$68,8 \quad(11)$ \\
$87,5 \quad(14)$ \\
$1,94(14)$ \\
$0 \quad(0)$ \\
68,8 \\
6,8
\end{tabular} & $\begin{array}{cc}17 \\
35,6 \quad(6) \\
64,7 \quad(11) \\
82,4 \quad(14) \\
2,19(14) \\
36, l^{4} \quad(4) \\
41,2 \\
5,4\end{array}$ \\
\hline 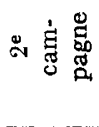 & $\begin{array}{l}\text { Nombre de femelles inséminées.. } \\
\text { Taux de non-retour } 110 \mathrm{j} \text { après la } \\
\text { dernière insémination (p. 100) }\end{array}$ & $\begin{array}{c}11 \\
36,4 \quad(4)\end{array}$ & $\begin{array}{c}15 \\
33,3 \quad(5)\end{array}$ & $73,9 \quad(17) !$ & $\begin{array}{l}1 ! \\
64,2 \quad(9)\end{array}$ & $6^{\prime} \pm, 2 \quad(9)$ \\
\hline
\end{tabular}

(1) Variable calculée pour les femelles ayant une date de naissance connue.

${ }^{(2)}$ Moyenne des deux contrôles.

( $\left.{ }^{3}\right)$ (...) figure l'effectif.

(4) Variable calculée sans tenir compte des accouplements en monte naturelle.

${ }^{(5)}$ Variable calculée en tenant compte des accouplements en monte naturelle. 
Blondes se sont avérées plus tardives que les Charolaises et croisées Charolaises. Cette observation avait également été faite dans l'expérience précédente. Nous avons de même observé par ailleurs (BIBÉ et al., I974a) que les femelles de la race Limousine proche de la race Blonde d'Aquitaine, étaient plus tardives que les Charolaises. Toutes ces conclusions concernant des génisses nées en fin d'hiver, doivent être interprétées en tenant compte non seulement de l'effet de l'âge mais aussi de différences saisonnières éventuelles dans les manifestations des chaleurs; il est à ce sujet frappant de constater sur la figure I que les races dont les signes de sexualité se manifestent précocement chez les génisses en fin d'hiver (Charolaise, Aubrac) sont également celles pour lesquelles les inséminations sont de loin les plus concentrées

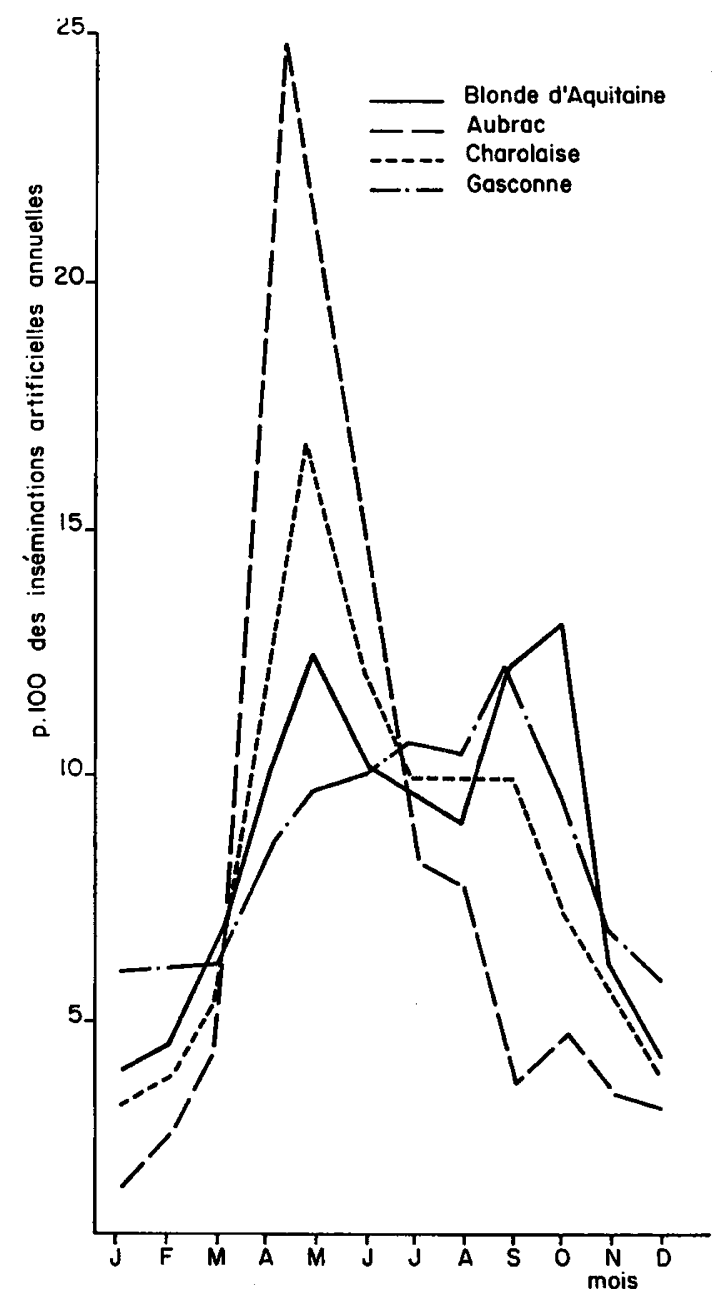

FIG. 1. - Répartition mensuelle des inséminations artificielles premières réalisées par

la coopérative d'élevage et d'insémination artificielle du sud-ouest, en 1968.

L'Aubrac et la Charolaise sont des races à vêlages groupés au printemps

La Gasconne et la Blonde d'Aquitaine sont des races à vêlages répartis sur l'année.

Monthly distribution of $1^{\text {st }}$ A.I. done by the A.I. Center (Coopelso) during 1968. 
à cette époque. Ce phénomène doit être lié au système d'exploitation des troupeaux : élevages extensifs avec vêlages de fin d'hiver pour les inséminations en Charolais et en $A u b r a c$, contre petits élevages pratiquant la production de veaux de boucherie avec des vêlages étalés sur l'année pour les races Gasconne et Blonde d'Aquitaine. On remarque enfin que les génisses croisées ont une précocité sexuelle supérieure en moyenne de 20 à 30 p. IOo à celle des génisses de races parentales.

\section{b) Fertilité (tab1. 2).}

Les taux de vêlage après la période d'insémination sont inférieurs à ceux relatifs à l'expérience réalisée sur femelles $A u b r a c$ (87 p. Ioo des femelles de l'essai " $A u b r a c$ " ont vêlé vers 2 ans contre 78 p. roo pour celles de l'essai « Gascon " qui n'ont d'ailleurs vêlé que vers 26 à 27 mois). Comme précédemment les taux sont plus élevés au I $^{\text {er }}$ vêlage pour les croisées Charolaises, au $2^{\mathrm{e}}$ vêlage pour les Blondes et croisées Blondes ; ces résultats peuvent être interprétés par la meilleure précocité sexuelle du Charolais et par l'incidence ultérieure défavorable des difficultés de vêlage des génisses, supérieures pour cette dernière race (voir plus loin).

Compte tenu du système d'élimination adopté, les troubles de reproduction ont eu pour conséquence une réduction considérable de la longévité : sur Ior génisses mises en expérience, 66 ont été gestantes après la période normale d'insémination (85 en tenant compte des résultats de la repasse en monte naturelle) 44 ont fourni un deuxième veau, une vingtaine seulement ont pu être conduites jusqu'au $3^{\mathbf{e}}$ vêlage. Ce sont les femelles Charolaises et croisées Charolaises qui ont été les plus affectées par cette élimination (moins de $25 \mathrm{p}$. Ioo subsistent pour le $2^{\mathrm{e}}$ vêlage), suivies par les Blondes et croisées Blondes (50 p. roo); 60 p. roo des femelles Gasconnes ont pu être conservées pour le second vêlage.

c) Aptitude au vêlage (tabl. 3).

Dans cette expérience, comparée à l'essai " $A u b r a c$ ", la fréquence des difficultés de vêlage était logiquement inférieure compte tenu, d'une part, du choix de taureaux donnant des veaux plus légers et, d'autre part, de l'âge plus tardif ( 2 à 3 mois) à la mise bas : I8 p. Ioo des vêlages ont eu lieu par césarienne au lieu de $30 \mathrm{p}$. Ioo, et 7 p. Ioo ont nécessité une aide difficile mais sans intervention chirurgicale contre 22 p. IOO.

De même, comme précédemment, les taureaux Charolais engendrent plus de difficultés (3I p. Ioo de césariennes et I5 p. roo de vêlages avec forte assistance) que les Blonds ( 3 p. Ioo de césariennes et ro p. Ioo de vêlages avec forte assistance). Cela est dû à la fois au poids de naissance plus élevé de leurs veaux $(+2,4 \mathrm{~kg})$ et à leur morphologie moins longiligne que celle des veaux Blonds. Rappelons que dans l'expérience sur femelles Aubrac et croisées, nous observions également des différences notables d'aptitude au vêlage entre races paternelles (45 p. Ioo de césariennes en Charolaise contre I9 p. Ioo en Blonde d'Aquitaine) pour des poids de veaux pourtant comparables à la naissance.

En ce qui concerne les types génétiques maternels (tabl. 2 et 3 ) les femelles Blondes et croisées Blondes fournissent les meilleurs résultats, les Charolaises et croisées Charolaises les plus mauvais ; de plus, le taux de vêlages difficiles des femelles croisées est en général plus faible que prévu compte tenu de l'aptitude au vêlage des races parentales. Ces résultats sont liés à divers phénomènes : 
- la préparation au vêlage (ABDALLAH et al., I97 I a) : elle semble mieux assurée chez les femelles Blondes et croisées Blondes pour lesquelles le relâchement des ligaments sacro-sciatiques intervient plus tôt et plus nettement. La tuméfaction de leur vulve est par contre moins accusée que celle des Charolaises et croisées Charolaises. Ce phénomène déjà observé dans l'expérience précédente et dans une comparaison entre les races Charolaise et Limousine proche de la Blonde (MÉNISSIER et al., I974 a), explique pourquoi les différences essentielles d'aptitude au vêlage entre femelles Charolaises et Blondes d'Aquitaine concernent surtout le taux de césariennes (indépendant de l'état de la vulve) et affectent peu le taux de vêlages difficiles.

- le déséquilibre morphologique fœeto-maternel : remarquons à ce sujet que si les poids de naissance augmentent du génotype Blond au Gascon et au Charolais (races pures et croisées envisagées séparément), l'ouverture pelvienne des femelles diminue elle simultanément (tabl. 3 et 7 ). Quant aux femelles croisées, 1'hétérosis qui semble se manifester sur leur format (poids vif et ouverture pelvienne) et sur le poids de naissance de leurs veaux (effet maternel) semble peu modifier l'équilibre entre les dimensions du veau à la naissance et celles de la mère.

\section{d) Viabilité des veaux.}

Les taux de mortalité au premier vêlage sont relativement plus faibles que ceux observés dans l'expérience sur génisses $A u b r a c$ et croisées. Nous n'avons enregistré aucun cas de mortalité dans la descendance des femelles croisées contre I 7 à $36 \mathrm{p}$. IOo pour celle des femelles de race pure : cela conduirait à penser à l'existence d'un effet d'hétérosis notable sur ce caractère, effet qui pourrait être en partie lié à la meilleure aptitude au vêlage des femelles croisées. Ce résultat comme les précédents devrait être vérifié sur un effectif d'animaux plus important.

Globalement le taux de veaux sevrés à la suite des inséminations des génisses est comparable en race Gasconne (65,5 p. I0o) et en race Aubrac (64,7 p. Ioo). Ce taux est nettement supérieur à celui relatif aux génisses de races à viande pures qui se situe autour de $40 \mathrm{p}$. Ioo. Les génisses croisées fournissent en définitive, grâce à la viabilité plus élevée de leurs veaux, les meilleurs résultats de productivité numérique (taux de veaux sevrés par femelles mises à la reproduction). Il est tentant de relier les résultats médiocres des races pures à ceux obtenus par CASU et al. (1975) sur les Charolaises conduites en zone de montagne. En effet, sans vouloir prétendre à un milieu aussi défavorable pour l'élevage des génisses dans le cas de notre expérience, il n'en reste pas moins qu'elles ont été soumises à un certain nombre de stress (alimentaires, déplacements) qui ont pu être ressentis différemment par les divers types génétiques maternels.

\section{2. - Croissance, comportement alimentaire, morphologie externe}

a) Phase avant sevrage (tabl. 3 et 4 ).

Les différences génétiques de croissance se manifestent surtout pendant la vie intra-utérine : elles traduisent essentiellement le potentiel supérieur de la race Charolaise par rapport à la Gasconne et à la Blonde d'Aquitaine, et ce malgré une durée de gestation plus longue pour les veaux issus de taureaux ou de femelles de ces deux dernières races. Notons que cet allongement de la durée de gestation se retrouve aussi 
dans la race Limousine (CASU et al., I975 ; CAVAGNE, I974) et semblerait donc une caractéristique du rameau Blond. Quant à la supériorité de poids de naissance des veaux issus de femelles croisées, elle peut être expliquée par une durée de gestation plus longue et surtout par un effet maternel positif lié au format plus élevé de ces femelles.

De la naissance au sevrage, la supériorité du potentiel de croissance des veaux issus de taureaux Charolais ne s'exprime pas en raison, probablement, d'une insuffisance de production laitière des mères et en l'absence de complémentation importante des veaux en concentré. En ce qui concerne l'effet du type génétique maternel, bien que les différences observées ne soient pas significatives, les veaux issus de vaches Charolaises et surtout croisées Charolaises se détachent par rapport à ceux issus de vaches Blondes. Cet avantage apparaît surtout à partir du $4^{\mathrm{e}}$ mois lorsque les veaux commencent à consommer directement et en quantité d'autres aliments que du lait. Jusqu'à cet âge, en effet, la croissance est comparable pour les veaux issus de femelles à viande et croisées, voire inférieure pour les veaux de mères Charolaises. Cela doit être rapproché des estimations de production laitière réalisées dans cet essai (tabl. 2) : les femelles Gasconnes apparaissent comme allaitant mieux leurs veaux que les femelles de races à viande ; les mères croisées sont en position intermédiaire.

L'étude du comportement du couple mère-veau et des variations de poids pendant la phase d'allaitement (tabl. 4) permet d'apporter quelques informations complémentaires :

- Les veaux issus de mères croisées tètent plus fréquemment que les autres en fin de lactation ; 1'hétérosis semble ainsi se caractériser, à ce stade, par un meilleur comportement maternel, et peut être par une plus grande persistance de la lactation (Schwulst et al., Ig68) ;

- Les femelles croisées dont le poids après vêlage est supérieur, accusent ensuite une perte de poids plus élevée, notamment pendant la période de sous-nutrition. Leur comportement alimentaire est voisin de celui des femelles Gasconnes ; il se caractérise tant avant (matin) qu'après (soir) la distribution de fourrages, par un appétit supérieur à celui manifesté par les mères de races à viande pures qui sont, par contre, observées plus fréquemment en état de rumination ;

- Par ailleurs, les femelles Blondes et croisées dont on a pu mettre en évidence, dans une autre expérience (VISINE, I973), la meilleure faculté d'adaptation au stress thermique, paraissent s'abreuver moins fréquemment que celles des autres types génétiques.

b) Phase d'engraissement (tabl. 5 et 6).

La supériorité de croissance des veaux issus de taureaux Charolais qui n'avait pu s'exprimer pendant la phase d'allaitement et qui n'apparaît pas non plus durant la saison de pâturage des génisses, se manifeste pleinement au cours de l'engraissement intensif ( +50 à $90 \mathrm{~g}$ de croissance journalière moyenne) et notamment dans les premiers mois de cet engraissement. Cela se traduit par une consommation journalière supérieure des croisés Charolais (+ 0,5 à $0,7 \mathrm{~kg}$ d'aliment par jour) ; l'indice de consommation étant comparable pour les veaux issus des deux races paternelles.

En ce qui concerne les types génétiques maternels, surtout chez les produits 


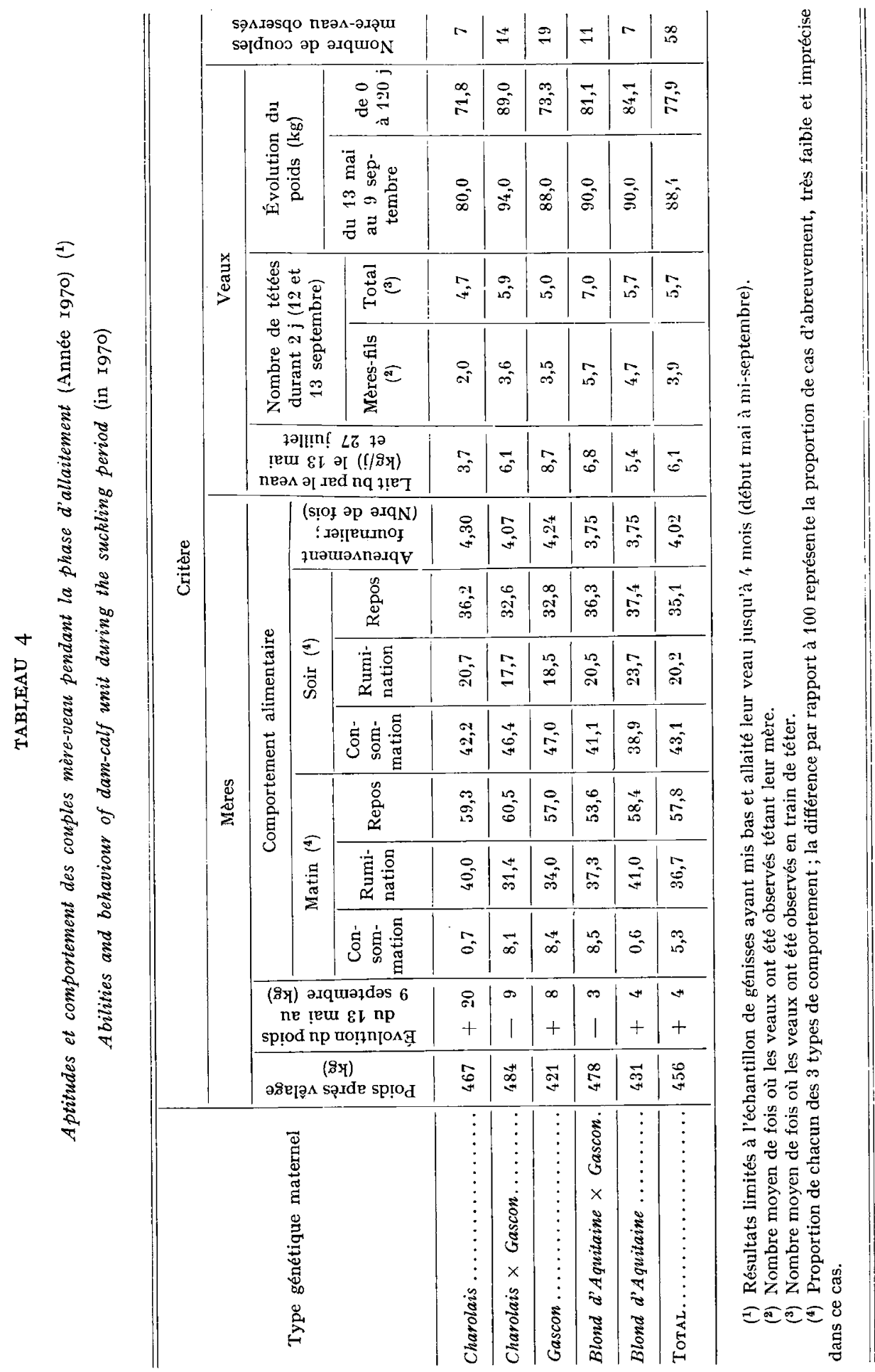


femelles, nous avons noté un avantage de croissance, d'une part, des veaux issus de mères à viande ou croisées par rapport à ceux nés de Gasconnes, d'autre part, des produits des mères croisées par rapport à la moyenne des produits des vaches des races parentales pures (hétérosis maternel). Ces différences de croissance correspondent à des écarts de poids final et de consommation alimentaire significatifs pour les veaux femelles. Il n'y a plus, globalement, de différences d'indice de consommation entre types génétiques maternels bien que les veaux issus de mères Blondes et croisées Blondes paraissent dans l'ensemble plus efficaces que ceux des autres types génétiques. Ce dernier résultat peut être expliqué essentiellement par une différence de composition corporelle, les veaux femelles issus de mères Blondes et croisées étant en moyenne plus maigres.

\section{TABLEAU 5}

Effets de l'année et du type génétique des veaux sur la croissance pendant l'engraissement : Produits mâles

Effects of calf's genotype and year on growth during fattening period : males

\begin{tabular}{|c|c|c|c|c|c|c|c|}
\hline \multirow{2}{*}{\multicolumn{2}{|c|}{ Facteur }} & \multicolumn{6}{|c|}{ Critère } \\
\hline & & Effectif & $\begin{array}{l}\text { Poids en début } \\
\text { engraissement } \\
(250 \mathrm{j})(\mathbf{1})(\mathrm{kg})\end{array}$ & $\begin{array}{l}\text { Poids fin } \\
\text { engrais- } \\
\text { sement } \\
\left.(432 \mathrm{j}){ }^{1}\right) \\
(\mathrm{kg})\end{array}$ & $\begin{array}{c}\text { Vitesse de } \\
\text { croissance } \\
(\mathrm{g} / \mathrm{j})\end{array}$ & $\begin{array}{c}\text { Consommation } \\
\text { moyenne } \\
\text { quotidienne } \\
\text { (kg d'aliment) }\end{array}$ & $\begin{array}{c}\text { Indice de } \\
\text { consommation } \\
\text { (kg aliment } / \mathrm{kg} \\
\text { gain) }\end{array}$ \\
\hline \multirow{3}{*}{$\underset{⿱}{\mathbb{Z}}$} & Moyenne ....... & 51 & 267,2 & 518,0 & 1378 & 9,48 & 6,87 \\
\hline & $\begin{array}{l}1970 \ldots \ldots \ldots \ldots \\
1971 \ldots \ldots \ldots \ldots \\
1972 \ldots \ldots \ldots \ldots\end{array}$ & $\begin{array}{r}33 \\
12 \\
6\end{array}$ & $\begin{array}{l}-12,0 \\
-10,5 \\
+\quad 22,5\end{array}$ & $\begin{array}{l}-25,2 \\
-13,4 \\
+38,6\end{array}$ & $\begin{array}{r}-73 \\
-16 \\
+89\end{array}$ & $\begin{array}{l}-0,69 \\
-0,06 \\
+0,75\end{array}$ & $\begin{array}{r}-0,144 \\
+0,137 \\
+0,007\end{array}$ \\
\hline & (F) $\ldots \ldots \ldots \ldots$ & - & $(2,45 \mathrm{NS})$ & $\left(4,58^{*}\right)$ & $(4,54 *)$ & $\left(4,81^{*}\right)$ & $(0,68 \mathrm{NS})$ \\
\hline \multirow{2}{*}{ 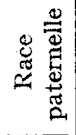 } & $\begin{array}{l}\text { Charolais ........ } \\
\text { Blonde d'Aquitaine }\end{array}$ & $\begin{array}{l}20 \\
31\end{array}$ & $\begin{array}{l}+\quad 1,5 \\
-\quad 1,5\end{array}$ & $\begin{array}{l}+\quad 6,5 \\
+\quad 6,5\end{array}$ & $\begin{array}{r}+28 \\
-28\end{array}$ & $\begin{array}{r}+0,26 \\
-0,26\end{array}$ & $\begin{array}{r}+0,077 \\
-0,077\end{array}$ \\
\hline & (F) $\ldots \ldots \ldots \ldots$ & 一 & 0,09 & $0,9^{\prime}$ & 2,43 & 2,75 & 0,55 \\
\hline \multirow[t]{2}{*}{ 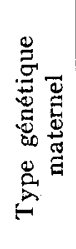 } & $\begin{array}{r}\text { Charolais ...... } \\
\text { Charolais } \times \text { Gas- } \\
\text { con ......... } \\
\text { Gascon ........ } \\
\text { Blond } \times \text { Gascon. } \\
\text { Blond d'Aquitaine. }\end{array}$ & $\begin{array}{r}1 / 4 \\
19 \\
10 \\
4\end{array}$ & $\begin{array}{l}-12,8 \\
+\quad 9,4 \\
-\quad 0,5 \\
-\quad 8,2 \\
-13,4\end{array}$ & $\begin{array}{r}7,7 \\
+\quad 16,7 \\
-\quad 1,8 \\
-\quad 5,9 \\
-16,8\end{array}$ & $\begin{array}{l}-27 \\
+40 \\
-7 \\
+13 \\
-19\end{array}$ & $\begin{array}{l}-0,19 \\
+0,66 \\
+0,22 \\
+0,01 \\
-0,69\end{array}$ & $\begin{array}{r}+0,018 \\
+0,301 \\
+0,238 \\
-0,101 \\
-0,456\end{array}$ \\
\hline & (F) $\ldots \ldots \ldots$ & - & $(0,66 \mathrm{NS})$ & $(0,62 \mathrm{NS})$ & $(0,44 \mathrm{NS})$ & $(1,45 \mathrm{NS})$ & $(1,23 \mathrm{NS})$ \\
\hline \multicolumn{2}{|c|}{ Interaction $\ldots \ldots \ldots \ldots$} & - & $(1,20 \mathrm{NS})$ & $(0,76 \mathrm{NS})$ & $(1,59 \mathrm{NS})$ & $(0,43 \mathrm{NS})$ & $(1,10 \mathrm{NS})$ \\
\hline \multicolumn{2}{|c|}{ Écart-type résiduel $\ldots}$. & - & 33,9 & 48,1 & 112,2 & 1,19 & 0,702 \\
\hline
\end{tabular}

(1) Age moyen, en jours.

NS : Effets non significativement différents au seuil de probabilité $p=0,05$.

* Effets significativement différents au seuil de probabilité $p=0,05$. 
B. BIBÉ ET COLL.

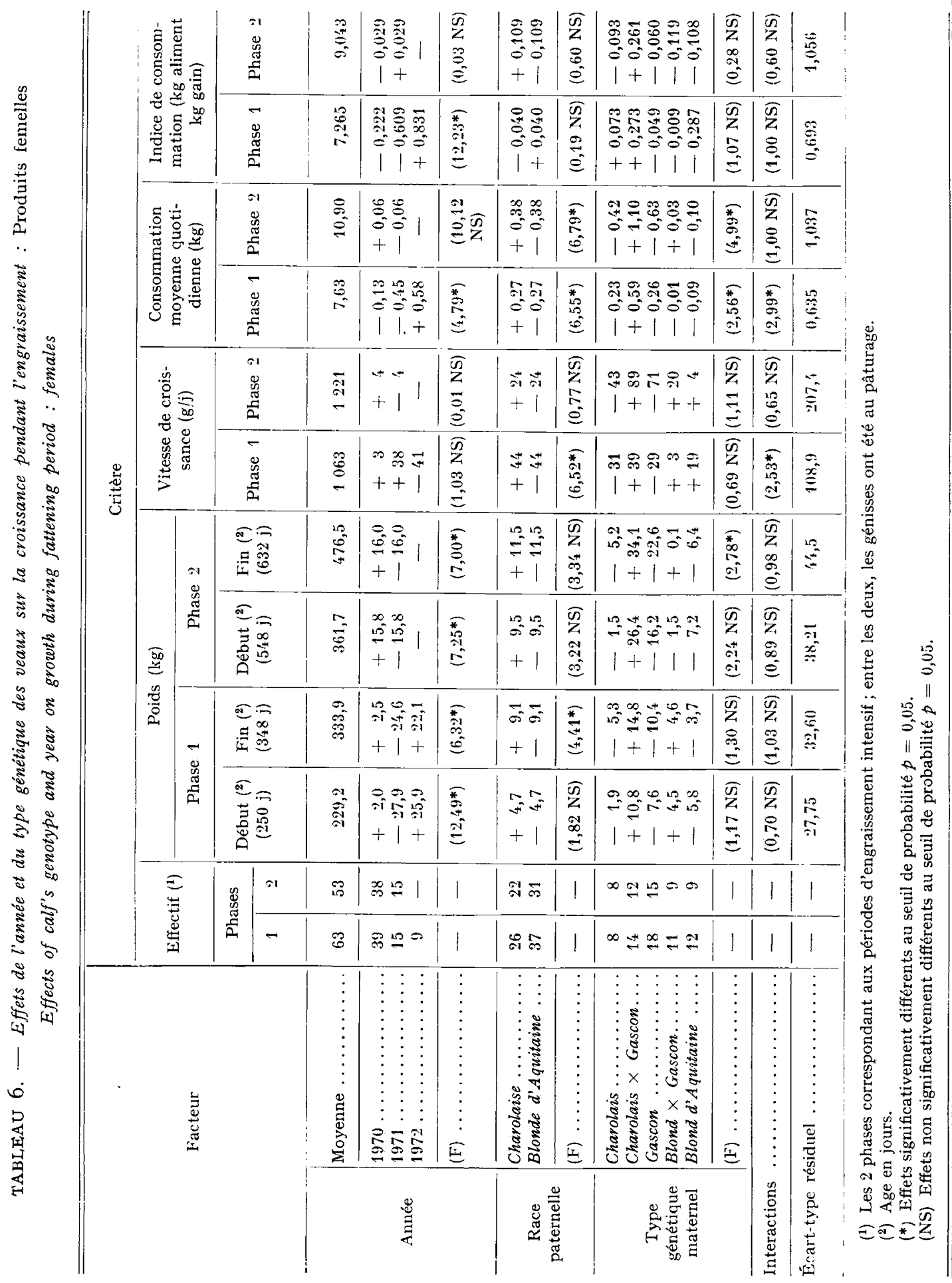


c) Morphologie externe (tabl. 7 et 8 ).

Les mères Charolaises pèsent en moyenne, dès l'âge de 2 ans, $50 \mathrm{~kg}$ de plus que les Blondes et les Gasconnes; les croisées ont un poids moyen supérieur à celui de chacune des deux races parentales. Les Charolaises sont plus compactes (taille inférieure pour une largeur aux trochanters maximum) ; les mères Blondes sont au contraire les plus grandes et les plus étroites au niveau des trochanters. Les différences de morphologie des veaux à un an entre types génétiques maternels et paternels, qui sont mises en évidence statistiquement pour la hauteur au garrot et la largeur aux trochanters, confirment les observations faites sur les mères : elles traduisent en effet l'existence d'un gradient de compacité croissante depuis les Blondes jusqu'aux Charolaises en passant par les Gasconnes et les croisées. Il est également intéressant de noter l'ouverture pelvienne très réduite des produits de mères de race à viande vis-à-vis de celle des produits issus de mères croisées et Gasconnes. Cela peut être rapproché de la meilleure aptitude au vêlage du premier groupe de mères. De même les veaux issus de vaches Charolaises et croisées Charolaises, malgré un poids plus

\section{TABLEAU 7}

Morphologie externe et composition corporelle des mères selon leur type génétique. Morphology and body composition of cows according to their genotype.

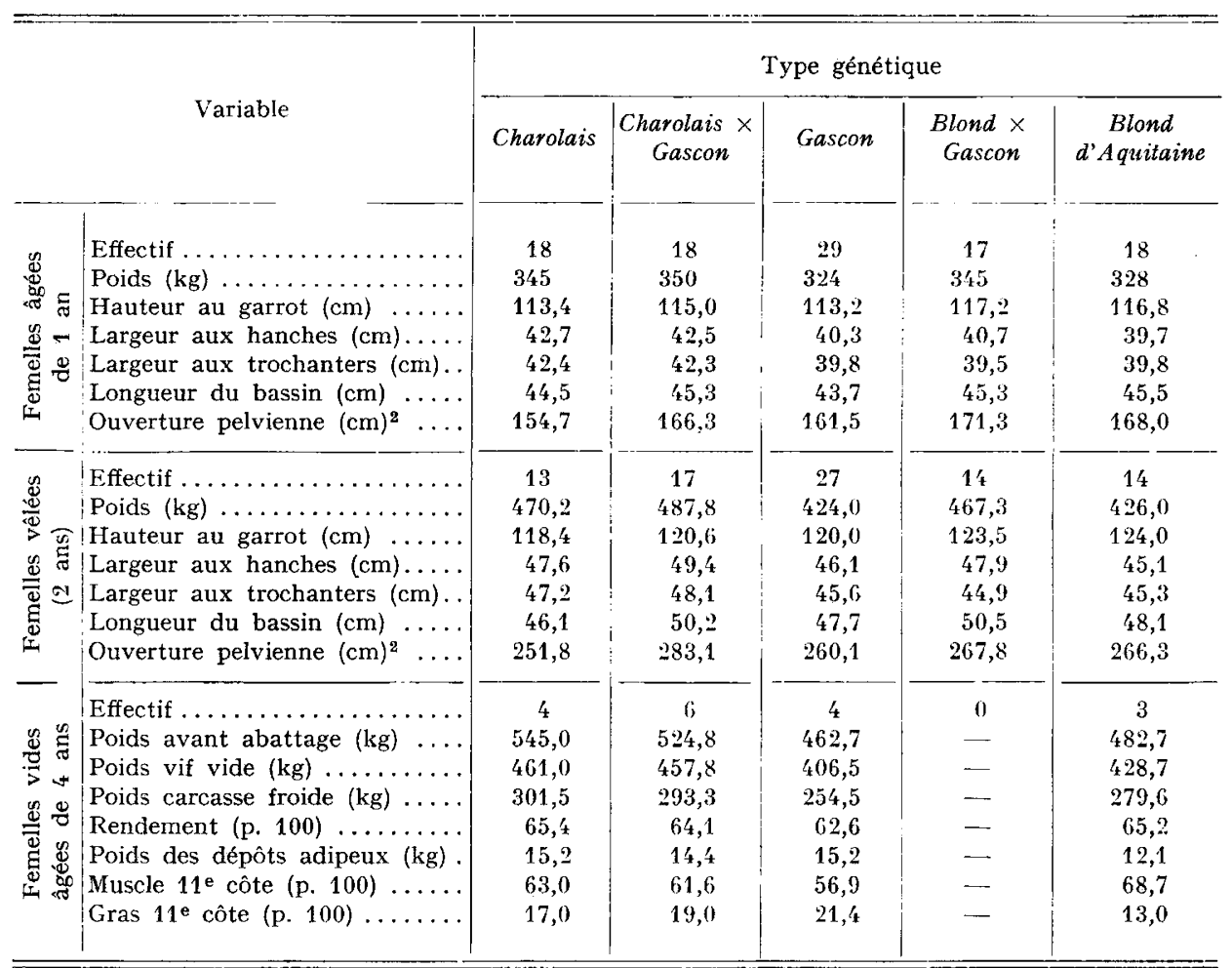


élevé que celui de leurs homologues Blonds, ont une ouverture pelvienne plus réduite ; ceci confirme, sur le plan morphologique, 1'aptitude au vêlage inférieure deș Charolaises par rapport aux Blondes.

\section{TABLEAU 8}

Effets de l'année, du sexe et du type génétique des produits sur leurs mensurations externes (1).

Effects of year, sex and genotype of calves on body measurements.

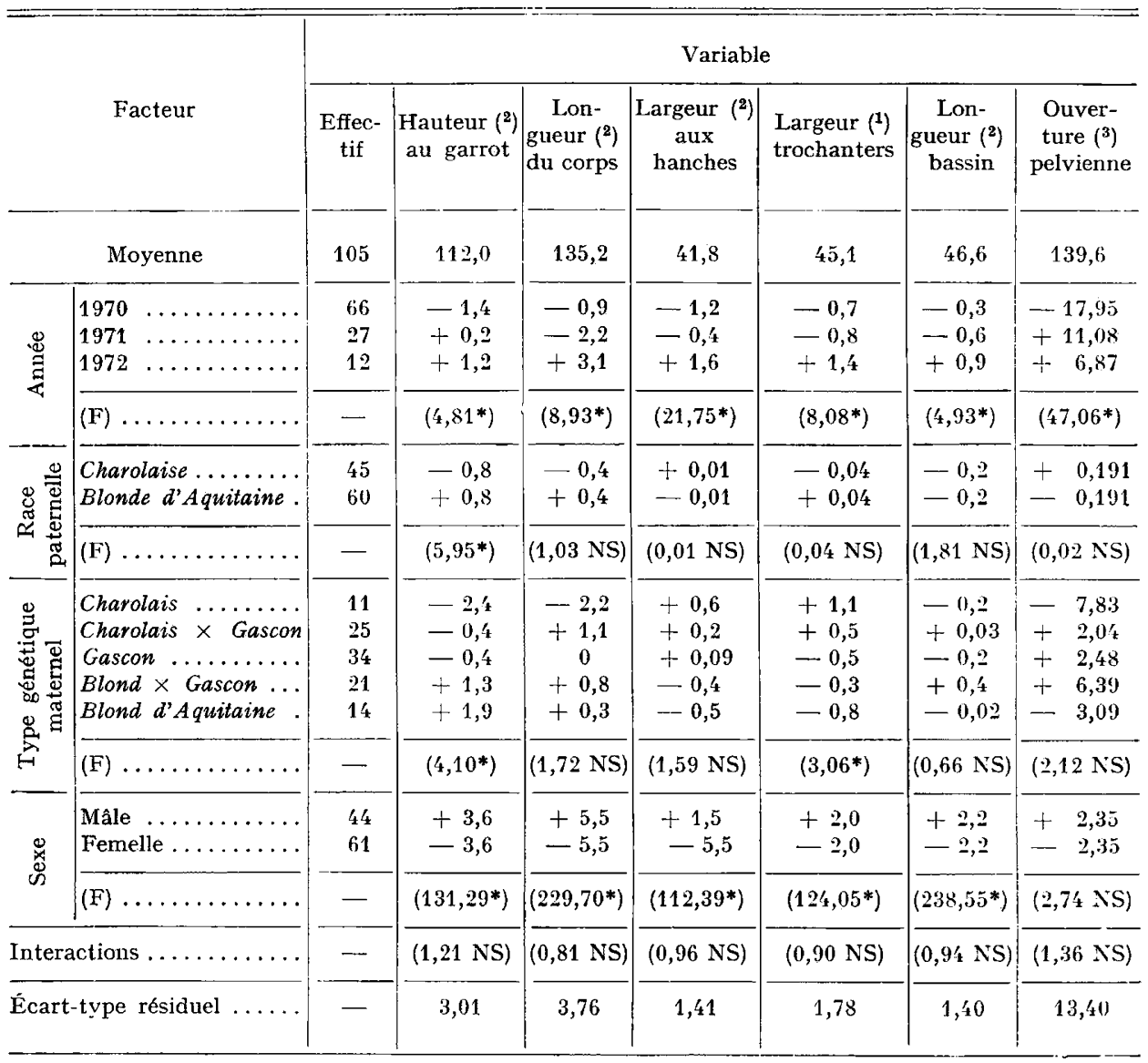

(1) Femelles mesurées à 12 mois, mâles mesurés à 13 mois; l'effet du sexe est confondu avec celui de l'âge à la mesure.

(2) En cm.

(3) En $\mathrm{cm}^{2}$.

(*) Effets significativement différents au seuil de probabilité $p=0,05$.

(NS) Effets non significativement différents au seuil de probabilité $p=0,05$. 


\section{3. - Qualités de carcasse et de viande}

a) Qualités de carcasse (tabl. 7, 9 et ro).

Nous avons enregistré un avantage de rendement à l'abattage $(+2$ à $+3 \mathrm{p}$. IOo) entre les mères à viande et les Gasconnes, ainsi qu'entre leurs produits respectifs (+ I à + I,5 p. I0o). Quant aux variations de poids de carcasse de ces derniers, elles traduisent de façon assez systématique la supériorité des produits des vaches et taureaux Charolais sur ceux des vaches et taureaux Blonds, ainsi que de ceux des vaches croisées sur ceux de chacune des deux races parentales.

Les dépôts adipeux sont plus réduits avec les types Blonds qu'il s'agisse des mères ou des produits mâles et femelles, et que l'on considère chez les produits les influences paternelles ou maternelles.

Cette adiposité plus réduite des animaux Blonds (vaches et produits purs et croisés) par rapport aux Charolais se retrouve au niveau de l'analyse du morceau unicostal. Par contre, bien qu'ayant des dépôts de gras interne comparables aux autres types génétiques, les vaches Gasconnes et leurs veaux fournissent les carcasses les plus grasses au regard du pourcentage de gras dans le morceau unicostal.

Quant au rendement en viande de la carcasse, il est nettement plus faible pour les vaches Gasconnes par rapport aux Charolaises $(-7$ p. Ioo pour les vaches, -4 à -5 p. Ioo pour leurs produits) et aux Blondes (- I 2 p. Ioo pour les vaches, -4 p. Ioo pour leurs produits). En ce qui concerne ces deux dernières races, l'avantage de la Blonde d'Aquitaine apparaît tant au niveau des carcasses des mères que de l'effet des pères sur la carcasse des produits ; les résultats sont plus irréguliers par contre pour l'effet du type génétique maternel sur le rendement en viande des produits.

Les variations des mensurations de carcasse entre génotypes sont surtout sensibles au niveau du membre postérieur : la cuisse des croisés Charolais est plus courte et plus épaisse que celles des croisés Blonds (types génétiques paternels et maternels) et Gascons (type génétique maternel).

b) Qualités de viande (tabl. II).

Les variations génétiques des caractéristiques de la viande n'interviennent essentiellement qu'au niveau de la couleur de la viande déterminée par la teneur en fer héminique. Les croisés Charolais (races paternelle et maternelle) donnent une viande plus colorée que les croisés Blonds d'Aquitaine; cependant ce sont les veaux issus de vaches Gasconnes et croisées qui donnent la viande la plus rouge. Un résultat équivalent avait été obtenu précédemment pour la race d'Aubrac vis-à-vis des deux mêmes races à viande. Pour les autres critères les différences n'apparaissent notables que pour les veaux mâles abattus à $I_{5}$ mois : la viande des veaux issus de vaches Gasconnes est plus dure et présente des pertes en eau inférieures à celles des produits de vaches de races à viande spécialisées. Il est intéressant de noter que ces variations génétiques sont beaucoup plus atténuées pour les carcasses des génisses abattues à 20 mois et ayant eu une période de pâturage. 


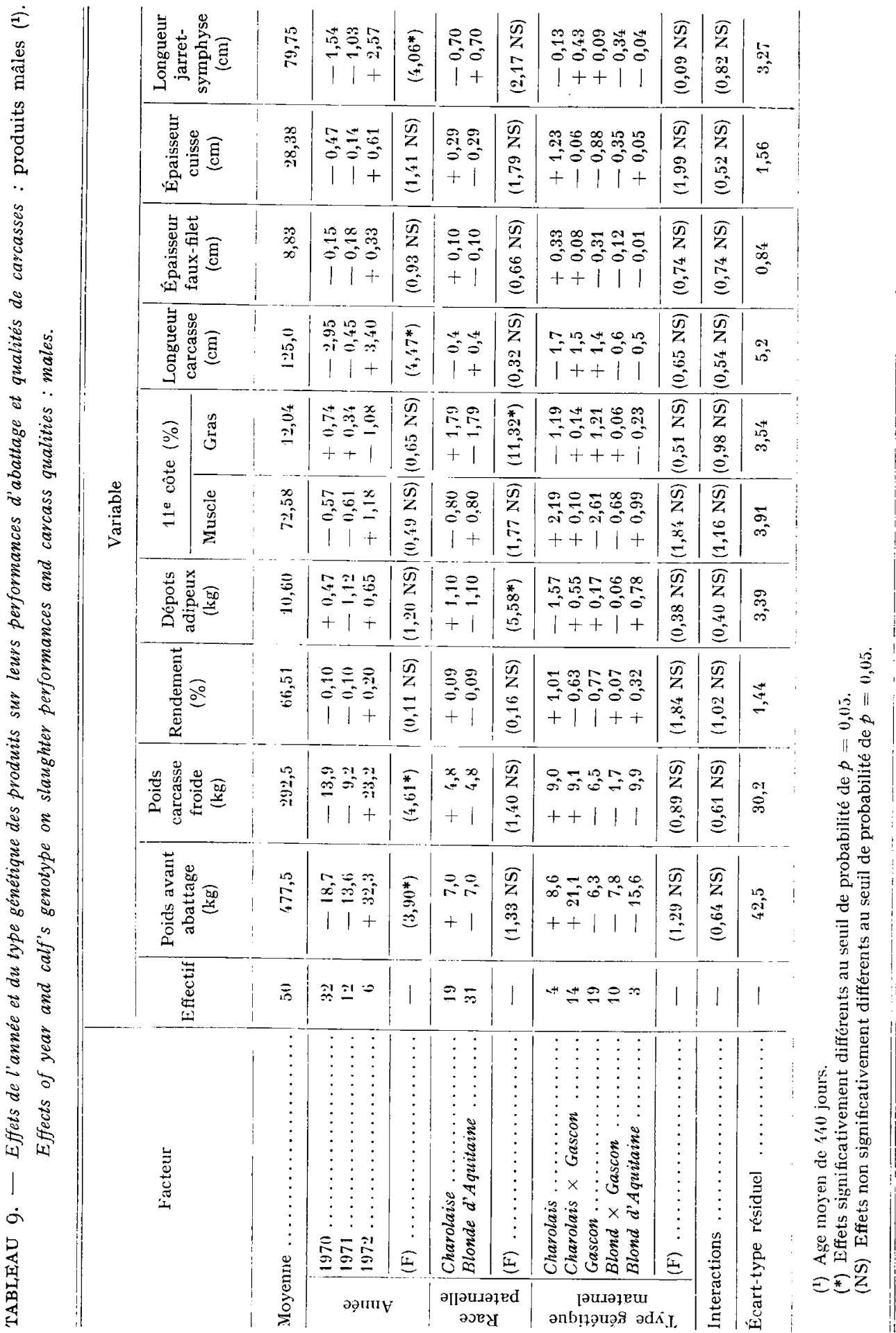




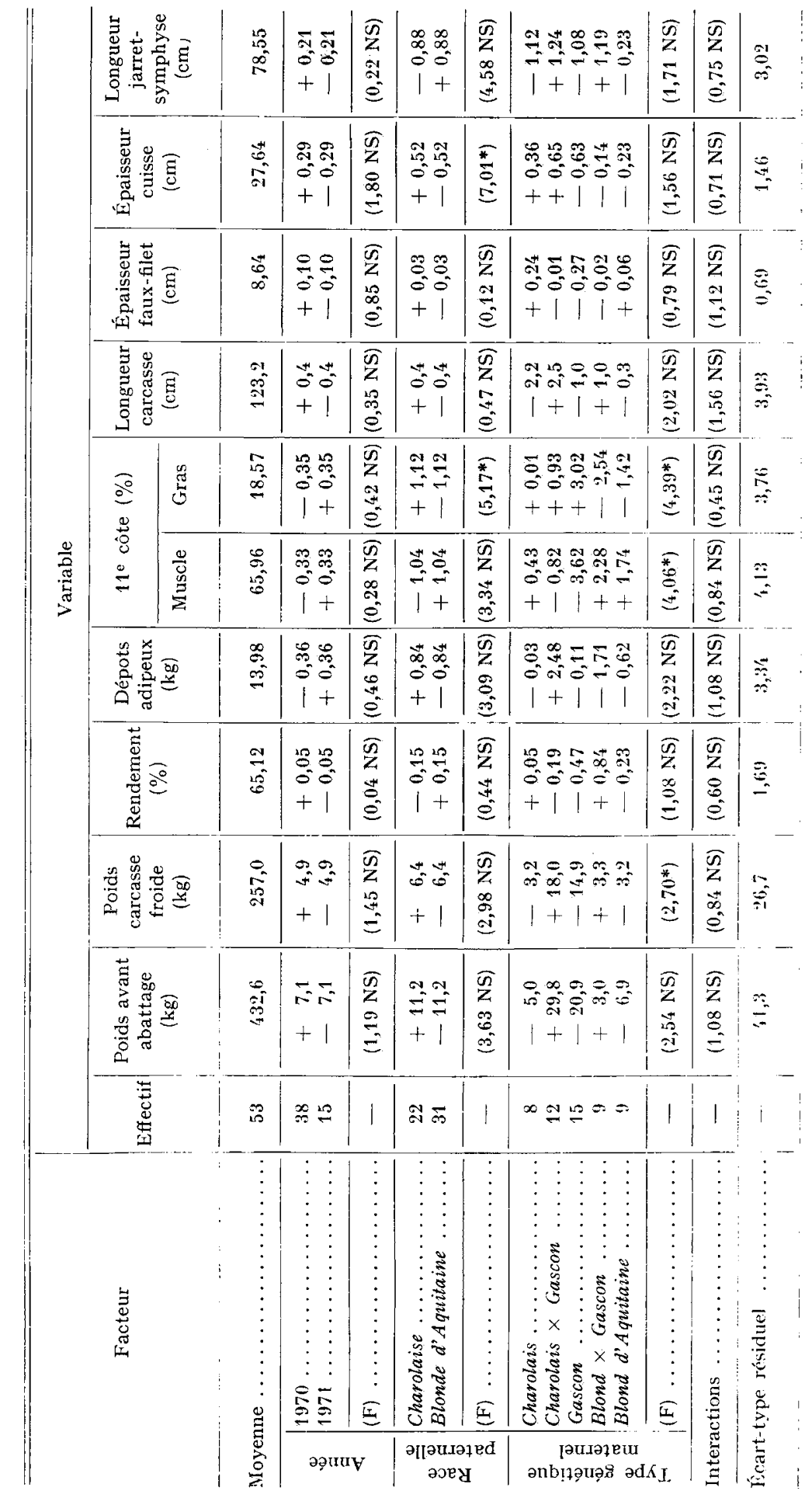




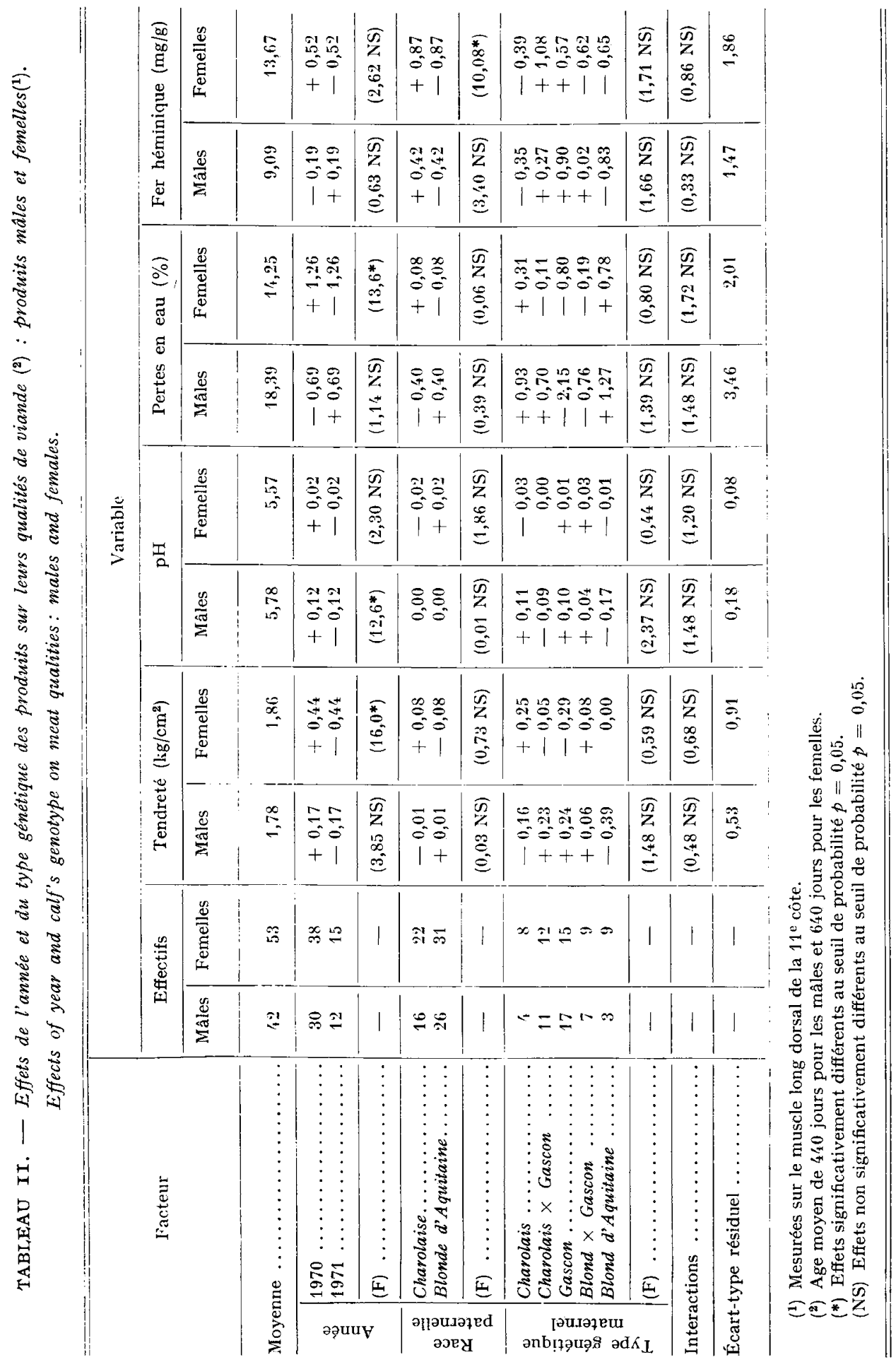




\section{DISCUSSION ET ANALYSE}

\section{I - Composantes de la variabilité génétique}

Il est apparu intéressant de compléter et d'approfondir la comparaison des résultats ci-dessus en estimant les composantes de la variabilité génétique, ce que le schéma adopté permet théoriquement de préciser. Il s'agit :

- des effets directs et maternels des races à viande pour les caractères influencés simultanément par les gènes du veau et de la mère ;

- des effets d'hétérosis, notamment, sur les effets maternels.

La signification de ces nouveaux résultats et leur interprétation dépend en fait de 1'homogénéité du matériel génétique représentant une même race aux divers niveaux où elle est considérée ; en particulier les races à viande interviennent :

Io comme race paternelle des veaux,

$2^{\circ}$ comme race paternelle des mères croisées Gasconnes,

$3^{\circ}$ comme races paternelle et maternelle des mères de race pure.

Cette condition d'homogénéité n'est pas totalement remplie; en particulier, le degré d'hypertrophie musculaire augmente vraisemblablement des mères des vaches de races à viande aux pères de ces vaches et des femelles croisées, ainsi que de ces derniers aux géniteurs nettement hypertrophiés qui ont été utilisés pour les inséminations. De plus, le degré d'accroissement de l'hypertrophie entre les catégories d'animaux a pu varier d'une race à viande à l'autre; il y a plus de chance pour que les pères des vaches utilisées dans l'expérience aient été préférentiellement de type culard en race Blonde d'Aquitaine (vaches à viande pures et croisées), à l'inverse de la race Charolaise. Il y a donc lieu de faire un certain nombre de réserves quant à la généralisation des résultats présentés ci-après.

\section{a) Effets direct et maternel des races à viande.}

Les valeurs obtenues pour ces effets sont rassemblées sur le tableau I2 pour les principaux caractères contrôlés dans cette expérience.

Les gènes $\mathrm{du}$ Charolais favorisent directement la croissance lorsque le milieu leur permet de s'exprimer (durant la vie utérine et les premiers mois de la phase d'engraissement par exemple) ; ils présentent également un effet maternel positif pendant la phase d'allaitement : ce résultat est plutôt surprenant compte tenu de la consommation plus faible des veaux issus de femelles Charolaises ou croisées (tabl. 4). Il pourrait être dû à une richesse supérieure du lait de ces femelles ainsi qu'à une alimentation non lactée plus précoce du veau Charolais dont l'aptitude à pâturer et ruminer précocement est souvent observée. Nous sommes par contre surpris de constater que cet effet maternel du Charolais sur la croissance, favorable pendant la phase d'allaitement, devienne négatif pendant l'engraissement : une meilleure adaptation des croisés Blonds au régime concentré leur permet peut être de rattraper le retard pris, avant sevrage, au pâturage auquel ils paraissent moins bien adaptés que les croisés Charolais. Remarquons que ces résultats sont opposés à ceux obtenus dans 1' " expérience $A u b r a c$ " en ce qui concerne d'une part l'évolution des effets direct et maternel avec l'âge des veaux, d'autre part le sens de ce dernier effet. 
B. BIBÉ ÉT COLL.

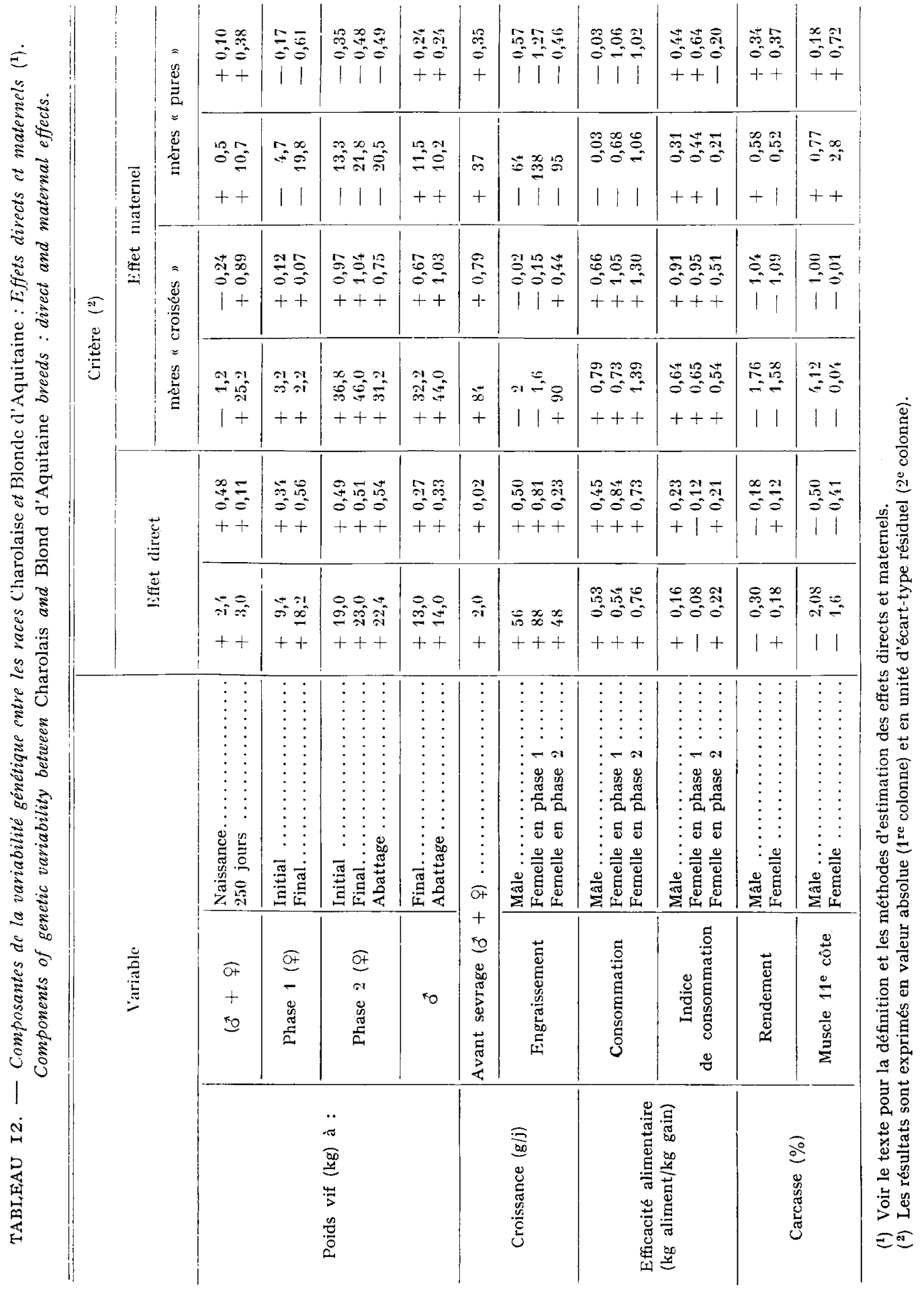


CROISEMENT AVEC LA RACE " GASCONNE "

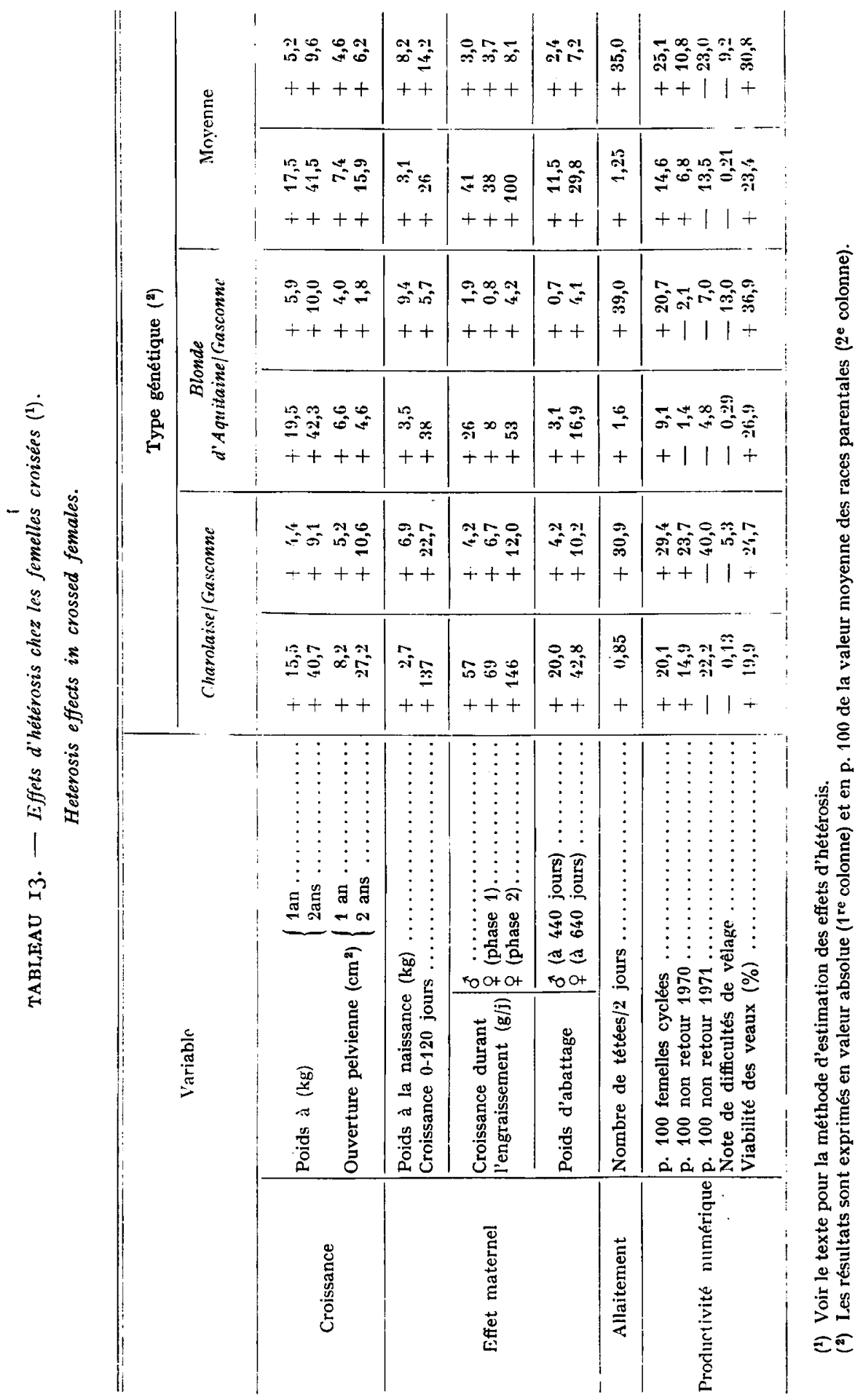

Annales de Génétique animale. — 1976. 
Les effets direct et maternel du Charolais se traduisent en moyenne par une augmentation de l'indice de consommation. Quant aux résultats relatifs au rendement et à la composition corporelle ils sont assez contradictoires ; ceci correspond peut-être à l'hétérogénéité des échantillons des reproducteurs de races à viande étudiés (voir ci-dessus).

\section{b) Hétérosis chez les femelles croisées (tab1. 13).}

Les valeurs de l'hétérosis estimées soit directement à partir des performances des femelles croisées, soit à partir des performances de leurs produits (hétérosis maternel) sont assez voisines de celles obtenues dans la littérature (CUNDIFF, I970 ; CuNDIFF et al., I974 $a$ et $b$ ). L'hétérosis varie de 5 à Io $\mathrm{p}$. Ioo pour la croissance des génisses. Il est comparable pour la taille de leurs ouvertures pelviennes et le poids à la naissance de leurs veaux, aussi est-il surprenant d'observer une valeur d'hétérosis favorable sur l'aptitude au vêlage (ro p. Ioo).

Les critères déterminant la productivité numérique sont les plus influencés par l'hétérosis qui intervient d'abord sur la précocité sexuelle $(25 \mathrm{p}$. Ioo) et la fertilité des génisses (II p. IOO), alors qu'au $2^{\mathrm{e}}$ cycle de reproduction, les mères croisées sont moins fertiles que celles des races parentales correspondantes (-23 p. IOO). Leur meilleure aptitude maternelle vers les $3^{\mathrm{e}}-4^{\mathrm{e}}$ mois de lactation (hétérosis de $35 \mathrm{p}$. Ioo sur le nombre de tétées) dans un environnement assez défavorable dont témoigne par exemple leur perte de poids en cours de lactation (tabl. 4), se solde probablement par un ralentissement de leur rythme de reproduction. C'est vraisemblablement cette bonne aptitude maternelle qui explique la meilleure viabilité des veaux des génisses croisées (hétérosis de $+26 \mathrm{p}$. Ioo en tant qu'effet maternel).

D'une façon générale, les valeurs d'hétérosis sont assez concordantes pour les deux comparaisons réalisées à partir des Charolaises et Blondes d'Aquitaine. Notons cependant les valeurs supérieures dans la première comparaison (Charolaises) ; ceci peut être rattaché au fait que la race Gasconne est génétiquement plus proche de la race Blonde d'Aquitaine que de la race Charolaise.

\section{2. - Bilans économiques}

Selon les formules décrites précédemment et à partir des paramètres estimés dans cette expérience, nous avons établi les divers bilans économiques pour chacun des types génétiques paternels et maternels.

\section{a) Races paternelles.}

Nous n'observons pas dans cette expérience de différences entre les deux races paternelles en ce qui concerne les résultats économiques du croisement terminal.

En effet les résultats inférieurs de la race Blonde d'Aquitaine sur la croissance (différence de poids de caracasse) sont compensés par une consommation légèrement plus faible et une proportion de muscle plus élevée.

Dans 1' " expérience $A$ ubrac " où nous avions observé sensiblement les mêmes écarts de croissance et consommation mais pas ceux relatifs au pourcentage de muscles, l'avantage était au croisement terminal avec le Charolais.

Dans ces deux expériences nous n'avons pas tenu compte des différences éven- 
tuelles entre les deux races paternelles concernant l'incidence des taureaux sur la fertilité de leurs inséminations ; le nombre de taureaux et les effectifs considérés étaient trop réduits pour les apprécier.

b) Types génétiques maternels.

Il ressort de 1'étude des différents bilans (tabl. I4) un certain nombre d'indications permettant de comparer l'efficacité de l'utilisation en croisement terminal des différents types génétiques maternels.

TABLEAU I4

Bilans économiques selon le type génétique maternel $\left({ }^{(}\right)$.

Economic results according to maternal genotype.

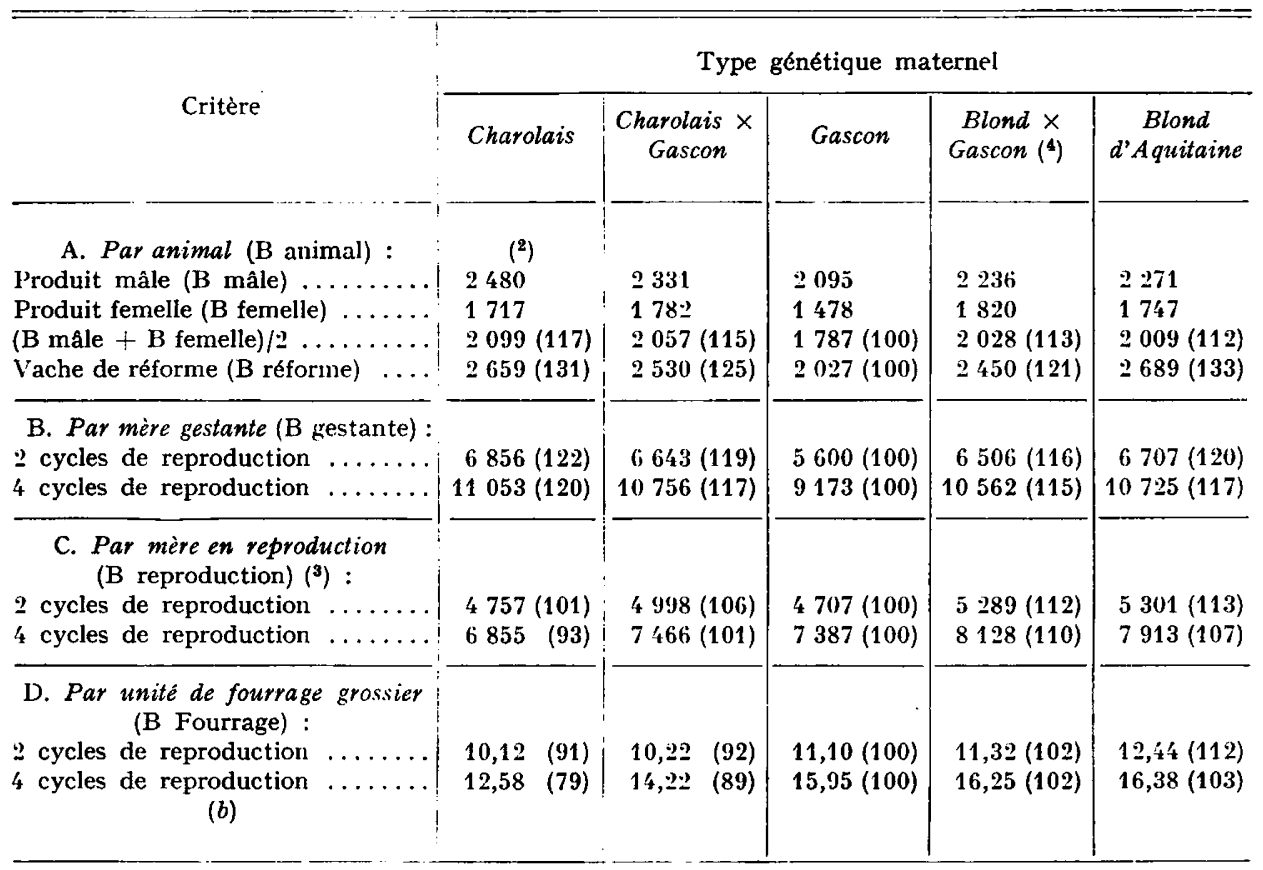

(1) Pour la définition et le mode de calcul de chaque bilan, voir le texte.

${ }_{(2)}{ }^{2}$ Les résultats sont exprimés en francs et en valeur relative par rapport à celle des Gasconnes pures (chiffres entre parenthèses).

$\left({ }^{3}\right)$ Nous avons retenu, d'après les résultats du tableau 2, les valeurs suivantes comme taux de veaux sevrés par femelle mise à la reproduction : 0,$50 ; 0,60 ; 0,75 ; 0,70 ; 0,65$ respectivement pour les types génétiques maternels mentionnés.

(4) Faute d'informations sur les performances d'abattage pour les vaches de réforme de ce type génétique, nous avons estimé le poids vif avant abattage à $500 \mathrm{~kg}$ et le poids de muscle à $175 \mathrm{~kg}$.

Si l'on ne prend pas en compte les différences de productivité numérique ou de besoins d'entretien liés au format des mères (bilans par mère gestante), ce sont bien évidemment les races à viande ou les croisées races à viande $\times$ race Gasconne qui l'emportent sur les femelles de race Gasconne pure ; leur supériorité moyenne sur 
cette dernière est voisine de $20 \mathrm{p}$. Ioo. Il tend a y avoir un léger avantage pour les Charolaises et croisées Charolaises par rapport aux Blondes d'Aquitaine et croisées Blondes.

La prise en compte des données de productivité numérique (bilan par mère en reproduction) bouleverse quelque peu ce classement. En effet selon l'écart enregistré pour cette variable entre la race Gasconne et le type génétique maternel considéré, cette différence subsistera ou non; la première situation est illustrée par les Blondes d'Aquitaine et croisées Blondes, la seconde par les Charolaises et à un degré moindre par les croisées Charolaises. L'impact de la productivité numérique sur ce classement est d'ailleurs d'autant plus grand que les mères sont supposées être utilisées pour un plus grand nombre de cycles de reproduction.

Enfin la considération du format des femelles dans ces bilans (bilan par unité de fourrages grossiers), accentue le désavantage des femelles Charolaises et réduit voire supprime (croisées Charolaises $\times$ Gasconnes) l'avantage des femelles croisées.

Il ne faut pas conclure trop hâtivement suite à l'analyse de ces premiers résultats, étant donné les effectifs et les conditions de réalisation de l'expérience; il faut cependant observer que dans les deux autres expériences comparables sur races rustiques ( $A u b r a c$ et $S a r d e$ ), nous avions trouvé une supériorité de productivité numérique des femelles de races rustiques sur celle des femelles croisées et celle des femelles de race à viande (Charolaise en Sardaign?).

Dans 1' "essai Aubrac " comme dans cette expérience, la différence n'était cependant pas telle qu'elle supprime l'intérêt de 1'utilisation de femelles croisées; par contre, en Sardaigne nous nous trouvions dans la situation inverse avec un avantage certain en faveur du simple croisement industriel.

\section{c) Schéma optimum de croisement.}

Nos résultats, avec les réserves qui viennent d'être faites, nous indiquent en premier lieu que la substitution de la race Gasconne par une race à viande spécialisée (cas du croisement d'absorption) n'est pas obligatoirement une solution économiquement intéressante.

Par contre, ils nous permettent d'envisager l'utilisation de femelles croisées comme reproductrices, avec peut-être une préférence à l'utilisation de taureaux Blonds d'Aquitaine pour les procréer. Quelle que soit la race paternelle, il sera de toute façon préférable d'utiliser des taureaux connus pour les qualités d'élevage de leurs filles plutôt que des taureaux à développement musculaire trop accusé sélectionnés pour le croisement terminal. Ensuite, on peut alors penser, soit à un croisement alternatif Blond d'Aquitaine, Gascon en utilisant à nouveau des taureaux Gascons sur ces femelles croisées (système conduisant à l'autorenouvellement du troupeau), soit à un croisement terminal avec abattage des produits mâles et femelles (système nécessitant le maintien d'un approvisionnement relativement important en femelles de race Gasconne pure).

Dans cette dernière situation, nos résultats ne nous permettent pas de choisir entre la race Charolaise et la race Blonde d'Aquitaine pour le croisement terminal ; mais si l'on voulait bénéficier du maximum d'hétérosis sur les produits, il conviendrait de choisir la race Charolaise. Toutefois, une conduite spéciale devrait être faite avec les jeunes femelles pour lesquelles l'utilisation de taureaux Charolais ne peut se faire qu'en admettant certains risques de difficultés de vêlages. 


\section{CONCLUSION}

Il est difficile pour différentes raisons de généraliser ces résultats obtenus sur un échantillon d'animaux très restreint. Les nombreux changements de localisation auxquels a été soumis le troupeau, faute d'une continuité suffisante dans l'attribution des moyens expérimentaux, doivent d'abord être invoqués pour expliquer le niveau moyen relativement médiocre de nombreuses performances de croissance et de productivité numérique. Nous avons par ailleurs extrapolé à un cycle vital, des résultats obtenus essentiellement sur de jeunes femelles; or, nous avons pu montrer que l'incidence des difficultés de vêlage, et du comportement maternel, pouvait modifier considérablement la fertilité des vaches des divers types génétiques telle qu'on 1'avait observée chez les génisses. En outre, cette expérience réalisée dans des conditions souvent artificielles sur des animaux spécialement contrôlés, n'a pu permettre d'étudier notamment le comportement des mères dans les systèmes d'élevages extensifs auxquels elles devraient être soumises en pratique. Or nous avons montré dans une expérience similaire réalisée en Sardaigne (CAsu et al., r975) dans de telles conditions, que d'autres facteurs non considérés ici pouvaient avoir une incidence importante sur la valeur respective des divers types génétiques :

- le comportement des taureaux, en monte naturelle, est particulièrement mauvais avec des taureaux à musculature hypertrophiée comme ceux utilisés dans cette étude par insémination artificielle. D'une façon générale nous manquons d'information sur le comportement sexuel des géniteurs mâles de ces différentes races ;

- l'adaptation des femelles de races à viande et même des croisées (viande $\times$ rustique et lait $\times$ rustique) à des restrictions alimentaires sévères est particulièrement médiocre surtout lorsqu'elles sont issues de taureaux culards. Dans de telles conditions, le potentiel laitier des femelles n'est souvent plus suffisant pour permettre l'extériorisation du potentiel de croissance des veaux croisés issus de mâles à viande. De ce fait, il n'apparaît pas justifié, dans de tels milieux, de développer les schémas de croisement au-delà de la première génération (CASU et al., 1975).

Compte tenu des résultats de cette étude et de ses limites, il a semblé justifié d'inciter la mise en place dans les fermes de la région pyrénéenne (Ariège, Aude, HauteGaronne), d'un schéma de croisement à double étage comportant l'utilisation de taureaux Blonds d'Aquitaine au premier niveau et de taureaux Charolais au deuxième niveau. Cette action comporte notamment l'organisation de l'élevage en vue d'assurer le maintien de femelles de race pure, l'utilisation de ces femelles pour le premier croisement, de celles des femelles $F_{\mathbf{1}}$ pour le croisement terminal. Elle vise également à vérifier et à compléter les résultats ci-dessus dans des milieux et avec des types de taureaux plus variés.

Ces recherches, comme les précédentes, devraient en fait déborder le cadre régional et génétique dans lequel elles ont été entreprises (Aubrac, Pyrénées, Sardaigne). Les races locales, dont elles mettent en évidence les potentialités pour l'élevage à viande extensif, devraient être comparées simultanément dans les divers milieux montagneux et méditerranéens dont elles sont originaires. Leurs caractères 
respectifs (adaptation à des restrictions alimentaires, production laitière, etc.) peuvent en effet se compléter en partie. L'étude plus globale sur une longue période des relations entre les facteurs du milieu, la végétation herbacée et arborée et le milieu humain, est une entreprise nécessaire pour préciser les conditions optima d'exploitation des zones concernées.

Ręu pour publication en avril 19i6.

\section{REMERCIEMEN'TS}

Nous tenons à remercier les nombreuses personnes qui ont participé à la réalisation de cette expérimentation ou qui ont contribué, par leurs critiques et suggestions, à la rédaction de ce mémoire.

\section{SUMMARY}

\section{USE OF HARDY BREEDS FOR CROSSING WITH BEEF BREEDS : EXAMPLE FOR THE GASCON BREED}

Since hardy breeds are not used any more for milking or draught they can be introduced into suckling herds subjected to industrial crossing with beef sires. The stock of these cows decreases rapidly ; it is therefore necessary to search for crossing methods giving highest profits, in extensive systems, from the natural resources of the areas where they are used. The present study concerns the Gascon breed widely spread over the Central Pyrenees, and completes previous findings obtained with the Aubrac breed as well as those gathered in Sardinia (Italy) with the Sarda and Modicana breeds.

The experimental herd was composed of about hundred heifers distributed into 5 breeding types : hardy breed, Gascon (GG), beef breeds, Charolais (CC) and Blond d'Aquitaine (BB) and crossbred heifers from sires of the 2 beef breeds $\times$ Gascon cows (CG and BG). During the experiment, the herd was kept in different localities under rather variable feeding conditions. The heifers calved at the age of 26-27 months and the herd was used for 3 years in terminal crossing with clouble-muscled Charolais and Blond d'Aquitaine sires. After weaning at about 7 months, all male and female progenies were intensively fattened and subjected to individual performance testing. They were slaughtered at the age of 14 months $1 / 2$ for the males after 182 days of fattening and at the age of 21 months for the females after two phases of intensive fattening ( 98 and 84 days) separated by a grazing period. Their carcasse were recorded.

The numerical productivity of the dam herd was analysed through the performances of the heifers. Primarily we observed a better sexual precocity in the crossbred heifers as compared to the parental breeds. In addition, heifers of Blond d'A quitaine dam (BB and BG) reached puberty later than heifers of Charolais dam (CC and CG). For that reason, calving rate of Charolais heifers was higher; on the other hand, at 2nd calving, pure and crossbred Blond d'Aquitainc heifers exhibited the best gestation rates, after Ist calving, the culling rate was high in Chavolais (more than 75 p. Ioo for CC and CG) as well as in Blond d'Aquitaine (about 50 p. Ino for BB and BG) and moderate in Gascon (about 40 p. Ioo for GG). The frequency of calving difficulties was lower in this herd of heifers than in the previous experiments : $25 \mathrm{p}$. 100 of difficult calvings ( 8 p. 100 of caesarian operations). Charolais sires induced more calving difficulties than Blond ones ( $3 \mathrm{I}$ and 3 p. Ioo, respectively of caesarian operations) as their calves were heavier and of less longshaped morphology. Blond d'Aquitaine dams exhibited less calving difficulties, on the one hand, because of a better balance between the weight of their calf and their pelvic opening, and, on the other, becattse of a good relaxation of their sacrosciatic ligaments. In the crossbred heifers, the heterosis affecting conformation and size as well as the weight of their calves, did not seem to modify their calving abilities. Mortality of the calves before weaning was high ( 77 to $36 \mathrm{p}$. roo) for straightbred clams, and inexisting for the crossbred ones. Thus, for a total of roo heifers, the Gascon dams weaned slightly fewer than the crossbred dams, but much more than the beef dams. 
Before weaning, calves from Charolais sires did not show any growth potential superiority probably because of insufficient milk production of the dams and creep-feeding of the calves. However, the calves of dams from Charolais (CC and especially CG) showed higher growth perfor-

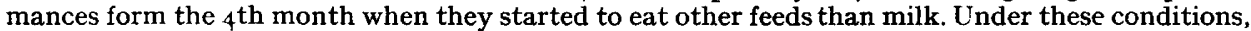
the Gascon appeared to be the most efficient nursing cows best. When observing the feeding behaviour of the dams and calves, during summer we noticed for the crossbred dams : I) that their calves suckled more frequently than the others 2) that their appetite after calving was similar to that of the Gascon dams and higher than that of the beef dams, and 3) that they seemed to ruminate less frequently. In addition, the heifers from Blond d'Aquitaine (BB and BG) did not drink as often as the others.

During fattening, the progeny of Charolais sires showed higher growth performances, but their feed conversion ratios were similar to those of Blond d'Aquitaine sires. The offsprings of Gascon dams exhibited lower growth performances. Maternal heterosis effects on growth were observed. Carcasses of progeny from Charolais (sires and dams) were the heaviest, whereas those from Blond d'Aquitaine were the leanest. Carcasses of Gascon cows and their progeny calves were the fattest despite the fact that their internal fat deposits were comparable to those the other genotypes; the meat yield of their carcasses was lower than that of Charolais ( -4 to $\cdots-7 \mathrm{p}$. Ioo) and Blond d'Aquitaine (-4 to - 12 p. Ioo). Charolais carcasses were more compact, notably in the hind-leg region. The meat of crossbred Chavolais was more colored than that of crossbred Blond d'Aquitaine; the offsprings from Gascon cows showed the most colored meat. As regards the other meat characteristics, we only found differences between breeding types in the male progeny (I 5 months) : the progeny of Gascon cows exhibited the toughest meat with the highest water retention.

An attempt was made to determine the differences between Chavolais and Blond d'.Aquitaine. breeds with respect to direct and maternal effects on growth : Charolais had a favourable direct effect on growth when kept under suitable environmental conditions; the maternal effect seemed to be positive before weaning and negative during fattening. Regarding the effects on body composition, the results were rather contradictory. We tried to determine the impact of heterosis on the main direct and maternal effects.

On the basis of these first parameters, we established economic balances for each paternal breed, maternal breeding type and crossing system. With the reserves depending on our parameters, we consider that the grading up of the Gascon breed to beef breeds is not justified. The use of crossbred dams for terminal crossing is probably the most profitable solution ; their replacement being realized either by alternative crossing (Blond-Gascon) or by keeping in pure a nucleus of Gascon dams for production of $F_{1}$ females.

It is difficult to generalize these conclusions drawn from a too small stock of animals mainly composed of young cows managed in a way not usual for that breed. Further research on a larger scale would be necessary to define more accurately the optimum utilization of the difficult environment by the hardy breed involved.

\section{RÉFÉRENCES BIBLIOGRAPHIQUES}

Abdallah O. Y., r971. Variations génétiques de l'aptitude au vêlage et de ses composantes. Thèse Doct. Ing. Fac. Sci., Paris, 300 p., bibliogr.

Abdallah O. Y., Hadjej M. S., MÉnissier F., I97I a. Variations du comportement pré et post-partum entre races rustiques, races à viande et croisement de première génération. Xe Congrès Int. Zootech., Paris-Versailles, juillet I971, 6 p.

ABdallah O. Y., MÉnissier F., Vissac B., I97I $b$. Liaison entre la musculature des races ct létr aptitude morphologique au vêlage: Résultats préliminaires. Xe Congrès Int. Zootech., juillet I971, ParisVersailles, $4 \mathrm{p}$.

Bibé B., Bonnet J. N., Cavagné S., Ménissier F., Sapa J., r974a. Comparaison des critères de productivité numérique et pondérale de trois races bovines à viande françaises : résultats partiels et préliminaires. Ann. Génét. Sél. anim., 7, 235-236.

Bibé B., Frébling J., MÉnissier F., 1974. Schéma d'utilisation des races rustiques er croisement avec des races à viande. VIe Journées du Grenier de Theix, "Le Troupeau de vaches allaitantes", novembre 1973, C.R.Z.V., Theix, Bull. tech. du C.R.Z.V., Theix (numéro spécial), 192-21 I.

Cartwright 'T. C., I970. Selection criteria for beef cattle for the future. J. Anim. Sci., 30, 706-7II.

Casu S., Boyazoglu J. G., Bibé B., Vissac B., I975. Systèmes d'amélioration génétique de la production de viande bovine dans les pays méditerranéens: Les Recherches Sardes. Bull. tech. Dép. Génét. anim. (Inst. nat. Rech. agron., Fr.) (22), pp. 56. 
CAVAGNÉ S., 1974. Étude comparative des résultats de reproduction de trois races bovines à viande . Rapport fin Études, Ec. nat. Ing. Trav. agric., Dijon, 88 p.

Cindiff 1. 1 ., r970. Experimental results on crossbreeding cattle for beef production. J. Anim. Sci., 30, 694-705.

CindifF L. V., GRÉgorY K. E., Koch R. M., 1974a. Effects of heterosis on reproduction in Hereford, Angus and Shorthorn cattle. J. Anim. Sci., 38, 71 I-727.

CundifF L. V., Grígory K. E., Schwulst F. J., Koch R. M., I974 $b$. Effects of heterosis on maternal performances and milk production in Hereford, Angus and Shorthorn. J. Anim. Sci., 38, 728-745.

Cunnixgham E. P., 1973. Cost-effectiveness and population structure in cattle breeding programs. Ann. Génét. Sél. anim., 5, 239-256.

Jickerson (i. E., r969. Experimental approaches in utilizing breed resources. Anim. Breed. Abstr., 37, I9I-202.

Dickerson G. E., r970. Efficiency of animal production-Molding the biological components. J. Anim. Sci., 30, 849-859.

Fơ lley J.-L., MÉnissier F., I974. Variabilité génétique des caractères de production de femelles Charolaises contrôlées en station: Résultats préliminaires. VI $\mathrm{e}$ Journées du "Grenier de Theix ". "L'exploitation du troupeau de vaches allaitantes ", novembre 1973 , Theix, Bull. tech., C.R.Z.V., Theix (numéro spécial), IクI-I9I.

Fréslini: J., Poujardiev B., Vissac B., Béranger C., Teissier J.-M., r967. Stations de sélection bovine. Compte rendu technique $\mathrm{n}^{\circ} \mathrm{I}$. Note générale. Bull. tech. Inf. (225), $8 \mathrm{p}$.

Gear Y., BÉranger C., r969. Estimation de la composition de la carcasse de jeunes bovins à partir de la composition d'un morceau monocostal ( I $^{\mathrm{e}}$ côte). Ann. Zootech., 18, 65-67.

Journé M. E., Legras P., Sornay J., r973. Méthodes d'étude de la qualité des caracasses et des viandes bovines. Inst. tech. Élev. Bov., 90 p. (ronéoté).

Ménissier F., Bibé B., Perreau B., i974a. Possibilités d'amélioration des conditions de vêlage par sélection. 11. L'aptitude au vêlage de trois races à viande françaises. Ann. Génét. Sél. anim., 6, 69-9o.

Ménissier F., Vissac B., I97r. Possibilités d'amélioration des conditions de vêlage par sélection. I. Technique de mesure de l'ouverture pelvienne des bovins. Ann. Génét. Sél. anim., 3, 207-214.

Ménissier F., Vissac B., Frébling J., I974. Plans optimaux de production bovine : l'amélioration génétique de la production de viande dans les troupeaux spécialisés et par croisement dans les troupeaux laitiers. Bull. tech. Dép. Génét. anim. (Inst. nat. Rech. agron., F.) (2I), "Optimum breeding plans for beef cattle "., 2-56 (in English). Ann. Génét. Sél. anim., 7, 229-230.

Schwelst F. J., Koch R. M., Grégorr K. E., Cundiff L. V., Sumption L. J., ig68. Heterosis of nilk production in beef cows. J. Anim. Sci., 27, 1129 (abstr.).

Visinf: J. L., I973. Adaptation des bovins à la chaleur. Rapport de fin d'Études, Éc. nat. Ing. Trav. agric., Bordeaux, ro8 p.

Vissac B., r97o. Étude génétique de la race d'Aubrac. In "l'Aubrac, étude éthnologique, linguistique, agronomique et économique d'un établissement humain ". C.N.R.S., Paris, 1, 27-IOI.

Vissac B., I972. 1. Sélection de souches mâles de bovins à viande pour le croisement terminal. In " Effficacité réelle et optimum du choix des taureaux de races à viande pour le croisement industriel ". Bull. tech. l'ép. (iénét. anim. (Inst. nat. Rech. agron., Fr.) (15), I-22. 